\title{
Design, Synthesis, And Evaluation of Functionalized Diamino-butylbenzamides As Selective Dopamine D3 Receptor Ligands
}

Benjamin E Blass ( $\sim$ benjamin.Blass@temple.edu )

Temple University School of Pharmacy https://orcid.org/0000-0003-2449-4503

Peng-Jen Chen

Temple University School of Pharmacy

Michelle Taylor

University of North Texas Health Science

Suzy A Griffin

University of North Texas Health Science

John C Gordon

Temple University School of Pharmacy

Robert R Luedtke

University of North Texas Health Science

\section{Research Article}

Keywords: Dopamine, D3 dopamine receptor, D2 dopamine receptor, substance use disorder, cocaine

Posted Date: October 19th, 2021

DOI: https://doi.org/10.21203/rs.3.rs-969699/v1

License: (1) This work is licensed under a Creative Commons Attribution 4.0 International License. Read Full License 
Design, synthesis, and evaluation of functionalized diamino-butylbenzamides as selective dopamine D3 receptor ligands

Benjamin E. Blass, ${ }^{\mathrm{a}}$ Peng-Jen Chen ${ }^{\mathrm{a}}$, Michelle Taylor ${ }^{\mathrm{b}}$, Suzy A. Griffin ${ }^{\mathrm{b}}$, , John C. Gordon ${ }^{\mathrm{a}}$, and Robert R. Luedtke ,

$\square$ Benjamin E. Blass

Benjamin.Blass@Temple.edu

Peng-Jen Chen

a18y270777@gmail.com

Michelle Taylor

michelle.taylor@unthsc.edu

Suzy A. Griffin

suzy.griffin@unthsc.edu

John C. Gordon

jackgordon@temple.edu

Robert R. Luedtke

robert.luedtke@unthsc.edu

${ }^{a}$ Temple University School of Pharmacy, Department of Pharmaceutical Sciences, 3307 North Broad Street, Philadelphia, PA 194140 USA

${ }^{\mathrm{b}}$ University of North Texas Health Science, Department of Pharmacology and Neuroscience, Center, 3500 Camp Bowie Blvd. Fort Worth, Texas 76107, USA 
Abstract: Substance use disorder remains a major, unmet medical need. Cocaine is one of the most commonly abused recreational drugs and in 2018, there were over 5.5 million current cocaine users. There are no approved therapies for the treatment of cocaine use disorder, but the D3 dopamine receptor has been identified as a potential therapeutic target. We have identified a series of functionalized diamino-butylbenzamides that are potent D3 binders with moderate to high selectivity for $\mathrm{D}_{3}$ over $\mathrm{D}_{2}$.

\section{Graphical Abstract:}

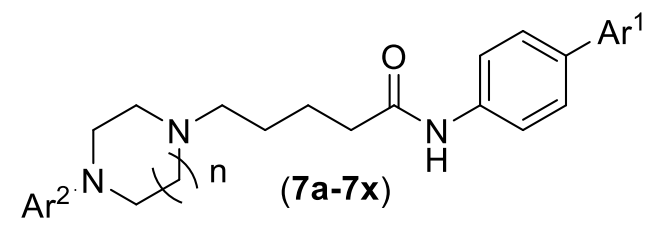

Keywords: Dopamine, $\mathrm{D}_{3}$ dopamine receptor, $\mathrm{D}_{2}$ dopamine receptor, substance use disorder, cocaine

Introduction: Substance use disorder (SUD) is a major societal and medical issue that a significant negative impact on SUD patients, as well as their family and caregivers. According to the 2018 National Survey on Drug Use and Health, approximately 32 million people over the age of 12 in the US reported consuming an illicit drug in the past 30 days, which equates to just over $19 \%$ of the US population. In addition, the US is home to over 8 million people suffering from addiction to illicit drugs such as cocaine (1), heroine, and methamphetamine. The estimated annual societal costs of SUD exceed $\$ 600$ billion,[1] and an increased risk of a range of conditions is definitively linked to SUD. Patients suffering from this condition experience increased rates of HIV and hepatitis infection, cardiovascular disease, lung disease, stroke, and cancer.[2]

Cocaine, which can be injected or snorted as the $\mathrm{HCl}$ salt or smoked as the free base (crack cocaine), is one of the most commonly abused drugs. Over 5.5 million people in the US 
described themselves as current user of cocaine in 2018[1]. The pharmacological impact of cocaine is partially driven by its ability to interact with dopamine transporters (DAT) in the brain and lower dopamine uptake. This interferes with the normal operation of the mesocorticolimbic dopamine (MCL-DA) system, which is more commonly known as the reward system.[3] Synaptic levels of dopamine rise as a result of cocaine's suppression of DAT activity, which leads to an amplification of dopamine signaling and creates the euphoric linked to cocaine abuse. As cocaine use continues, changes in DAT and dopamine receptors occur, which causes longterm neuroadaptation of several brain circuits.[4] Chronic exposure to cocaine is also linked to negative impacts on executive function and decision-making processes, as well as increased risks of emotional affect such as anxiety, panic, paranoia, and violent behavior. Patient attempting to end their use of cocaine experience withdrawal symptoms including serious deficits, mainly in working memory, planning, and cognitive flexibility.[5] Cocaine overdose is also a serious risk for patients, and scale of this issue in the US was outlined in the 2011 Drug Abuse Warning Network report. This study found that in 2011, >500K patients required emergency room services as a result of cocaine overdose.

The magnitude of this problem is clear, but unfortunately therapeutic options are limited. In some patients, behavioral interventions such as contingency management programs provide rewards for abstinence is effective,

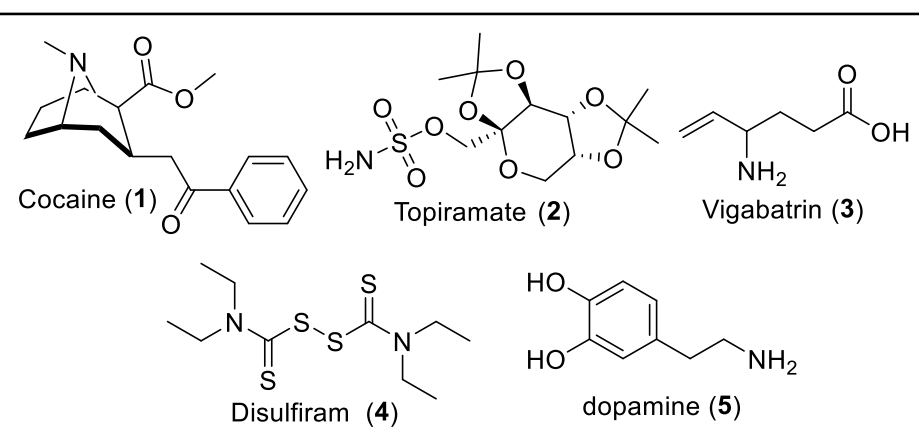

Figure 1: Structures of Cocaine (1), Topiramate (2), Vigabatrin (3), Disulfiram (4), and Dopamine (5).

while others respond to cognitive-behavioral therapies that teach recovering addicts to recognize 
and avoid situations that will trigger the desire to use cocaine. These methods are, however, far too costly and resources intensive to meet the needs of the patient population in need of treatment.[7] In principle, behavioral interventions and cognitive-behavioral therapies could be paired with a suitable pharmacological intervention, but there are no FDA approved medications for the treatment of cocaine addiction, also known as cocaine use disorder (CUD). Research efforts focused on this issue have led to exploration of novel compounds, as well as effort to repurpose previously approved therapies.[8] The AMPA/Kainate receptor antagonist Topiramate (2)[9] and the antiepileptic irreversible inhibitor of GABA transaminase Vigabatrin (3),[10] for example have also been examined in clinical trials, but neither have been approved for use as treatments for cocaine addiction. The alcoholism treatment Disulfiram (4) has shown promise in a limited patient population, but its mechanism of action is unknown.[11] Positive results in animal studies with compounds that selectively target the neurokinin-1 receptor,[12] the cysteine glutamate antiporter,[13] and the 5-HT2C serotonin receptor [14] have also been reported.

In an effort to develop novel therapies with the potential to address the needs of patients suffering from CUD, we have focused our efforts on the possible positive impact of modulating dopamine (5) signaling. This neurotransmitter is

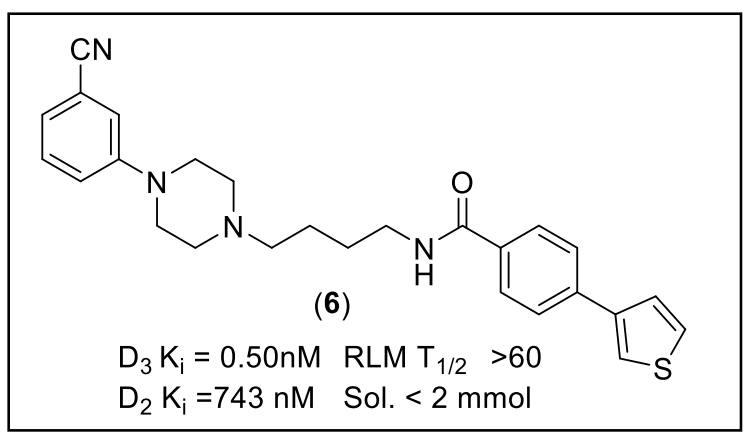
synthesized in the brain and periphery. In the periphery dopamine (5) can act as a vasodilator, modulate renal sodium excretion, and impact urine output, while in the central nervous system, dopamine (5) has a significant impact on movement, behavioral motivations, and learning.[15] The pharmacological impact of dopamine is mediated by a family of related G-protein coupled receptors (GPCRs) which are designated as designated $\mathrm{D}_{1}, \mathrm{D}_{2}, \mathrm{D}_{3}, \mathrm{D}_{4}$, and $\mathrm{D}_{5} .$. There are two 
sub-families of dopamine (5) receptors, the $\mathrm{D}_{1}$-like sub-family ( $\mathrm{D}_{1}$ and $\left.\mathrm{D}_{5}\right)$ and $\mathrm{D}_{2}$-like subfamily $\left(D_{2}, D_{3}\right.$ and $\left.D_{4}\right)$. These classifications are based on pharmacological properties, amino acid homology, and genetic organization.[16] Multiple lines of evidence suggest that $\mathrm{D}_{3}$ may be a viable therapeutic target for the treatment of CUD. Chronic and acute exposure to cocaine, for example, leads to increased $D_{3}$ expression in the nucleus accumbens. This area of the brain is responsible for cognitive processing including behaviors associated with CUD.[17] In addition, $\mathrm{D}_{3}$ is highly expressed in key brain regions that have been definitively linked to cocaine addiction. The role of $\mathrm{D}_{3}$ in CUD has been described in multiple review articles.[18] There is also evidence implicating $\mathrm{D}_{2}$ signaling as a possible therapeutic target for the treatment of CUD, but there are significant risks associated with $\mathrm{D}_{2}$ modulation (e.g. extrapyamidal symptoms, catalepsy).[19] In order to avoid these potential risks, we have focused our efforts on the development of novel $\mathrm{D}_{3}$ ligands that are highly selective for $\mathrm{D}_{3}$ over $\mathrm{D}_{2}$.

We have recently described the identification of a series of $\mathrm{N}-(4-(4-$ phenyl piperazin-1yl)butyl)-4-(thiophen-3-yl)benzamides that bind to $\mathrm{D}_{3}$ selectively over $\mathrm{D}_{2}$. As part of these studies, we identified (6) as a highly potent $\mathrm{D}_{3}$ ligand $\left(\mathrm{K}_{\mathrm{i}}=0.5 \mathrm{nM}\right)$ that demonstrated a significant selectivity for $\mathrm{D}_{3}$ over $\mathrm{D}_{2}\left(\mathrm{~K}_{\mathrm{i}}=743 \mathrm{nM}\right)$.[20] As part of our broader effort to understand the source of $\mathrm{D}_{3} / \mathrm{D}_{2}$ selectivity, we determined that while $\mathrm{D}_{3}$ and $\mathrm{D}_{2}$ binding are

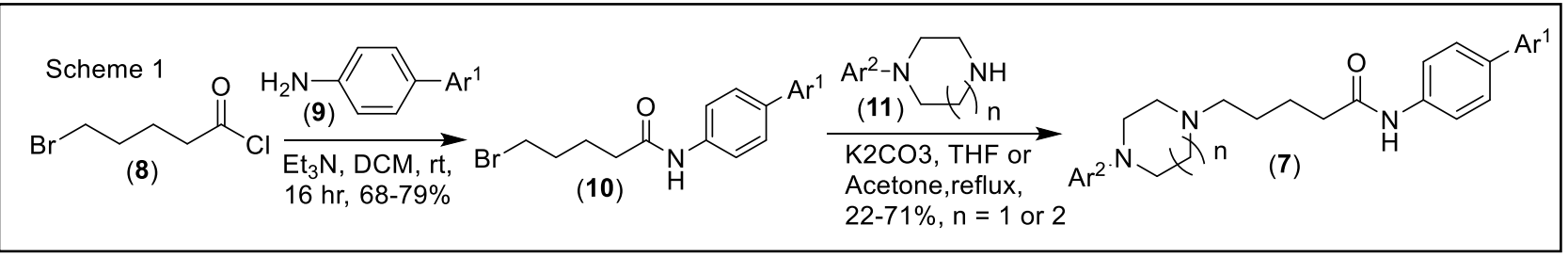

driven primarily by the aryl piperazine portion of the molecule (via $\mathrm{Asp}^{3.32}$ ), $\mathrm{D}_{3} / \mathrm{D}_{2}$ selectivity is primarily driven by the phenyl thiophene region.[21] Preliminary rat pharmacokinetic studies revealed that $(6)$ has a long terminal $\mathrm{T}_{1 / 2}(10.9 \mathrm{~h})$, but solubility studies revealed that this 
compound is poorly soluble in aqueous media ( $\mathrm{Sol}<2 \mathrm{mmol}$ ). While there are examples of poorly soluble, FDA approved medication, we viewed this as issue that should be addressed in next generation compounds. We have therefore continued our efforts to identify novel, selective $\mathrm{D}_{3}$ ligands.

Results and Discussion: Our recent efforts have focused on a related series of compounds (7) in which the amide of (6) has been inverted. These compounds are readily prepared using the chemistry described in scheme 1 . In this process, 5-bromopentanoyl chloride (8) is reacted with a suitably substituted aniline (9) in the presence of triethyl amine to provide amide (10). Displacement of the primary bromide with a functionalized cyclic diamine (11) in refluxing acetone and in the presence of potassium carbonate provide the final screening targets. Table 1 describes the in vitro screening results for D3 and D2, as well as solubility and rat liver microsomal stability.

In our first series of compounds (7a-7i), we maintained the thiophen-3-y moiety in order minimize the number of changes between our new series and compounds typified by (6). As noted in table 1 , electron donating (7a) and election withdrawing groups $(\mathbf{7 b}, \mathbf{7 c})$ in the 2 poistion provide potent $\mathrm{D} 3$ binders $(\mathrm{Ki}=3.1 \mathrm{nM}, 6.2 \mathrm{nM}$, and $20 \mathrm{nM})$ with moderate selectivity over D2 (21-40 fold). However, rat liver microsome (RLM) stability of these compounds was low to moderate (19.3 to 5.6 minutes). Potent $\mathrm{D}_{3}$ activity was also observed when electron withdrawing groups were located in the 3-position, but selectivity over D2 was not uniform. Specifically, while the 3-CN analog (7d) was highly selective for D3 (D3 Ki=14.9 nM, D2 Ki = $8119 \mathrm{nM})$, the 3-CF3 analog $(7 \mathbf{e})$ demonstrated similar D3 binding $(\mathrm{Ki}=5 \mathrm{nM})$ but was far less selective for D2 $(\mathrm{Ki}=82.1 \mathrm{nM})$. RLM stability for both $(\mathbf{7 d})$ and $(\mathbf{7 e})$ was high $(\mathrm{T} 1 / 2=60 \mathrm{~min})$. Dichlorination $(\mathbf{7 f}, \mathbf{7 g}$, and $\mathbf{7 h})$ also provided potent D3 binders $(\mathrm{D} 3 \mathrm{Ki}=2-44 \mathrm{nM})$ that 
displayed moderate selectivity over D2 (108 to 392-fold), and were highly stable in the presence of RLM ( $\left.\mathrm{T}_{1 / 2}=60 \mathrm{~min}\right)$. Solubility of this initial set of analogs $(\mathbf{7 a - 7 f})$ was low.

Now that we had established that $\mathrm{D}_{3}$ activity could be retained when the central amide was inverted, we turned our attention towards identifying structural changes that might improve solubility. We initially examined the impact of replacing the thiophen-3-y ring with a 2-pyridine ring $(\mathbf{7 i}-\mathbf{7 0})$ while maintaining previously employed aryl substituents. With the exception of the 2,4-di-Cl (7o) and 3,5-di-Cl (7p), solubility improved (184-10.2 mM). Importantly, D 3 binding potency was high $(4.5 \mathrm{nM}-36 \mathrm{nM})$ and selectivity over $\mathrm{D}_{2}$ was moderate to high (20 to 277 fold). RLM stability was low when the when substituents were placed in the 2-position of the aryl piperazine $(7 \mathrm{i}-7 \mathrm{k}$ RLM T1/2 $=3.7$ to $7.7 \mathrm{~min})$, but improved when electron withdrawing substituent were place in the 3-poistion $(71,7 \mathrm{~m} \mathrm{RLM} \mathrm{T1/2}=10.9,20.9 \mathrm{~min}))$ or when the aryl piperazine contained 2 chlorine substituents (7n-7p, RLM T $1 / 2=15.1,18.2,60 \mathrm{~min}$.$) .$

We also explored relacing the central piperazine with a homopiperazine as a method of increasing solubility while maintaining $\mathrm{D}_{3}$ potency and selectivity. Examples (7q) to (7x) incorporate this piperazine bioisostere, preserve the thiophen-3-y ring, and employ the same sat of aryl substituents on the aryl piperazine ring. As noted in table 1, D3 potency and selectivity decreased when the piperazine is replaced by a homopiperazine. Solubility of the monosubstituted aryl piperazine analogs $(\mathbf{7 q - 7} \mathbf{u})$, but no significant improvement in solubility was observed with the dechlorinated analogs $(\mathbf{7 v}-\mathbf{7 x})$. High RLM stability was observed when that aryl piperazine unit was substituted with $3 \mathrm{CF}_{3}(7 \mathrm{u}$ RLM T1/2 $=24.1 \mathrm{~min}), 2,4-\mathrm{di}-\mathrm{Cl}(7 \mathrm{w}$, $\left.\mathrm{RLM} \mathrm{T}_{1 / 2}=44.5 \mathrm{~min}\right)$, and 3,5-di-Cl $\left(7 \mathbf{x}, \mathrm{RLM} \mathrm{T}_{1 / 2}=43.2 \mathrm{~min}\right)$. 
Conclusion: In summary, we have identified a new series of potent D3 binders that display moderate to high selectivity over D2. In addition, exemplary compounds have high RLM stability and aqueous solubility within an acceptable range. Additional efforts will be focused on further optimizing the physicochemical properties of this series of compounds while maintaining the $\mathrm{D}_{3} / \mathrm{D}_{2}$ selectivity observed in better performing compounds.

Experimental methods and materials: Reagents were purchased from Fisher Scientific, VWR International, Sigma Aldrich, and Combi-Blocks, Inc. Chromatographic purification of compounds (normal phase and reverse phase) was carried out on a Teledyne Isco Combiflash RF system. H-NMR spectra were obtained on a Bruker 400-MHz NMR. Chemical shift values $(\delta$ values) were reported in ppm relative to TMS. For multiplicity, $\mathrm{s}=\operatorname{singlet}, \mathrm{d}=$ doublet, $\mathrm{t}=$ triplet, $\mathrm{m}=$ multiplet. Purity (\%) and mass spectral data were determined with a Waters Agilent $1200 \mathrm{HPLC} / \mathrm{MS}$ (Zorbax SB-C18, 2.1 x $30 \mathrm{~mm}, 3.5 \mu \mathrm{m}, 100 \%$ water/ $0.1 \%$ formic acid to $100 \%$ acetonitrile $/ 0.1 \%$ formic acid over 4.0 minutes, $1.0 \mathrm{~mL} / \mathrm{min}$.) with a diode array detector from 210-400 nm and Agilent 6130 quadrupole MS. All compounds were purified to 95\% purity or greater as determined by HPLC/MS and 1H-NMR. Melting points were recorded on a capillary melting point apparatus.

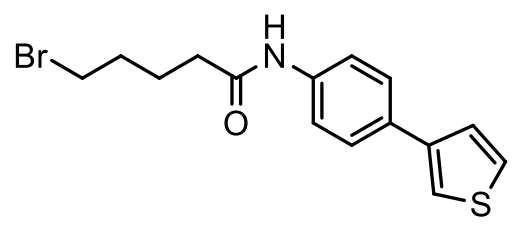

Preparation of 5-bromo-N-(4-(thiophen-3-yl)phenyl)pentanamide (10a): The 4-(thiophen-3yl)aniline (0.42 g, $2.36 \mathrm{mmol})$ was stirred with 5-bromovaleryl chloride $(0.77 \mathrm{ml}, 2.60 \mathrm{mmol}, 1.1$ eq) in the presence of anhydrous $\mathrm{N}, \mathrm{N}$-diisopropylethylamine $(0.5 \mathrm{ml}, 5.20 \mathrm{mmol}, 2.2 \mathrm{eq})$ in anhydrous dichloromethane for 16 hours. After 16 hours, the reaction mixture was stripped of 
solvent and the residual material was purified by normal phase chromatography, eluting with $40 \%$ dichloromethane in hexane to provide 5-bromo-N-(4-(thiophen-3yl)phenyl)pentanamide.(640mg, 79\%). ${ }^{1} \mathrm{H}$ NMR $\left(400 \mathrm{MHz}, \mathrm{CDCl}_{3}\right) \delta 8.01(\mathrm{~s}, 1 \mathrm{H}), 7.88(\mathrm{~d}, 1 \mathrm{H}, \mathrm{J}$ $=3.2 \mathrm{~Hz}), 7.78(\mathrm{~d}, 2 \mathrm{H}, \mathrm{J}=7.6 \mathrm{~Hz}), 7.65(\mathrm{~d}, 2 \mathrm{H}, \mathrm{J}=7.4 \mathrm{~Hz}), 7.42(\mathrm{~d}, 1 \mathrm{H}, \mathrm{J}=3.1 \mathrm{~Hz}), 3.49(\mathrm{t}, 2 \mathrm{H}$, $\mathrm{J}=6.8 \mathrm{~Hz}), 2.39(\mathrm{t}, 2 \mathrm{H}, \mathrm{J}=7.1 \mathrm{~Hz}), 1.79(\mathrm{~m}, 2 \mathrm{H}), 1.59(\mathrm{~m}, 2 \mathrm{H}) . \mathrm{MS}\left(\mathrm{LC} / \mathrm{MS}, \mathrm{M}+\mathrm{H}^{+}\right): 339.55$

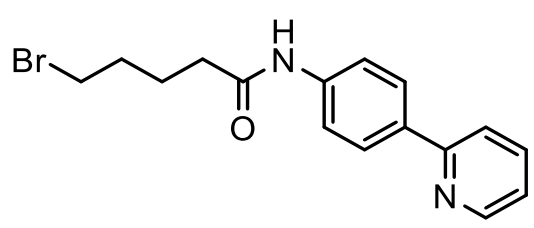

Preparation of 5-bromo-N-(4-(pyridin-2-yl)phenyl)pentanamide (PJC07-109) (10b): A solution of 4-(pyridin-2-yl)aniline $(0.1955 \mathrm{~g}, 1.15 \mathrm{mmol})$ and 5-bromovaleryl chloride $(0.23 \mathrm{ml}, 1.72$ mmol, 1.5 eq) in anhydrous dichloromethane was stirred at room temperature. Anhydrous triethylamine $(0.42 \mathrm{ml}, 2.99 \mathrm{mmol}, 2.6 \mathrm{eq})$ was added dropwise into the reaction at $0{ }^{\circ} \mathrm{C}$. The reaction was warmed to room temperature and stirred for 16 hours. After 16 hours, the reaction mixture was stripped of solvent and the residual material was purified by normal chromatography (100\% dichloromethane to $80 \%$ ethyl acetate in dichloromethane) to provide 5bromo-N-(4-(pyridin-2-yl)phenyl)pentanamide (261 mg, 68\% yield). ${ }^{1} \mathrm{H}$ NMR (400 MHz, $\left.\mathrm{CDCl}_{3}\right) \delta 8.35(\mathrm{~d}, 1 \mathrm{H}, \mathrm{J}=7.2 \mathrm{~Hz}), 8.28(\mathrm{~d}, 2 \mathrm{H}, \mathrm{J}=7.4 \mathrm{~Hz}), 7.75(\mathrm{~d}, 2 \mathrm{H}, \mathrm{J}=7.4 \mathrm{hZ}), 7.41,(\mathrm{~m}$, 1H), $7.12(\mathrm{~d}, 1 \mathrm{H}, \mathrm{J}=6.9 \mathrm{~Hz}), 6.95(\mathrm{~m}, 1 \mathrm{H}), 3.49(2 \mathrm{H}, \mathrm{J}=7.1 \mathrm{~Hz}), 2.42(\mathrm{t}, 2 \mathrm{H}, \mathrm{J}=6.8 \mathrm{~Hz}), 1.78$ (m, 2H), $1.65(\mathrm{~m}, 2 \mathrm{H}) . \mathrm{MS}\left(\mathrm{LC} / \mathrm{MS}, \mathrm{M}+\mathrm{H}^{+}\right): 334$. 


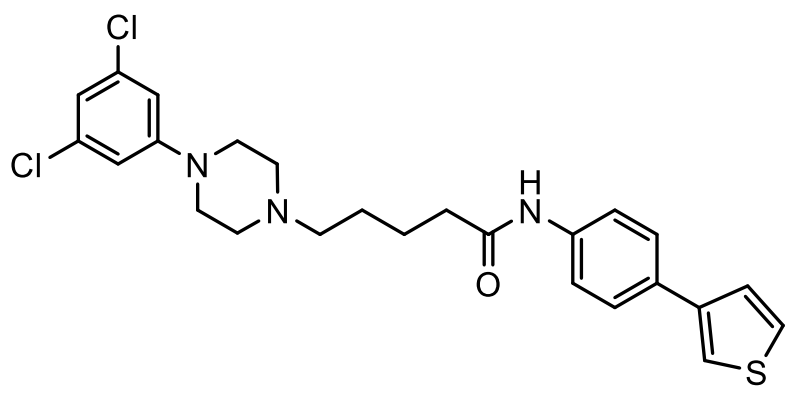

Preparation of 5-(4-(3,5-dichlorophenyl)piperazin-1-yl)-N-(4-(thiophen-3-yl)phenyl)pentanamide (MC25-67; PJC03-54) (7h): A solution of 5-bromo-N-(4-(thiophen-3-yl)phenyl)pentanamide (0.35 g, $1.03 \mathrm{mmol}), 1$-(3,5-dichlorophenyl)piperazine (0.48 g, $2.06 \mathrm{mmol}$, $2.0 \mathrm{eq})$ and potassium carbonate $(0.43 \mathrm{~g}, 3.09 \mathrm{mmol}, 3.0 \mathrm{eq})$ in dry tetrahydrofuran $(6 \mathrm{ml})$ was refluxed at $66^{\circ} \mathrm{C}$ for 48 hours. After 48 hours, the mixture was cooled and filtered to obtain a filtrate. The filtrate was purified by normal phase chromatography $(10 \%$ methanol in dichloromethane) to give a partial pure product. The impure product was further purified by reverse phase chromatography (100\% acetonitrile/0.1\% ammonium hydroxide) to give 5-(4-(3,5ichlorophenyl)piperazin-1-yl)-N-(4-(thiophen-3-yl)phenyl)pentanamide (242 mg, $48 \%$ yield). ${ }^{1} \mathrm{H}$ NMR (400 MHz, $\left.\mathrm{CDCl}_{3}\right) \delta 7.49(\mathrm{~s}, 3 \mathrm{H}), 7.33(\mathrm{~m}, 1 \mathrm{H}), 7.30-7.29(\mathrm{~m}, 2 \mathrm{H}), 6.73(\mathrm{t}, \mathrm{J}=1.68,1.56$ $\mathrm{Hz}, 1 \mathrm{H}), 6.66(\mathrm{~d}, \mathrm{~J}=1.68 \mathrm{~Hz}, 2 \mathrm{H}), 3.42(\mathrm{~d}, \mathrm{~J}=5.52 \mathrm{~Hz}, 2 \mathrm{H}), 3.17($ broad s, 4H), 2.56 (broad s, $3 \mathrm{H}), 2.42($ broad s, 2H), $2.36(\mathrm{t}, \mathrm{J}=7.32,7.2 \mathrm{~Hz}, 2 \mathrm{H}), 1.75$ (pent, 2H), $1.59(\mathrm{~m}, 2 \mathrm{H}) ; \mathrm{MS}$ (LC/MS, M+H'): 488.55 .

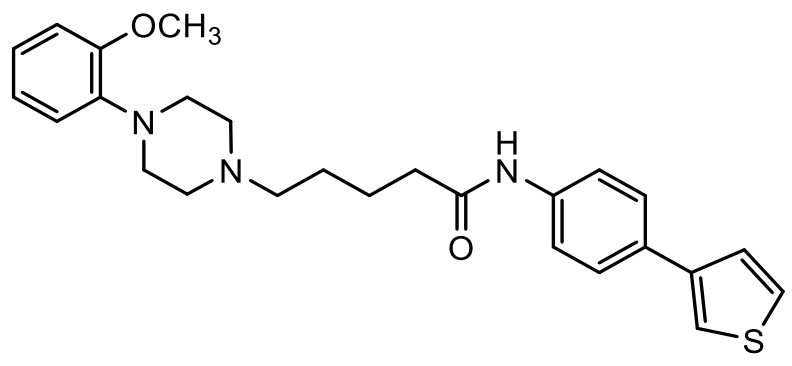

Preparation of 5-(4-(2-methoxyphenyl)piperazin-1-yl)-N-(4-(thiophen-3-yl)phenyl)Pentanamide (7a): The title compound was prepared according to the procedure for 5-(4-(3,5- 
dichlorophenyl)piperazin-1-yl)-N-(4-(thiophen-3-yl)phenyl)pentanamide, $\quad$ except $\quad 1-(2-$ methoxyphenyl)piperazine was substituted for 1-(3,5-dichlorophenyl)-piperazine. The product was first purified by normal phase chromatography (10\% methanol in dichloromethane) and was then further purified by reverse phase chromatography (30\% acetonitrile/ $0.1 \%$ formic acid in water) to provide 5-(4-(3,5-dichlorophenyl)piperazin-1-yl)-N-(4-(thiophen-3yl)phenyl)pentanamide (32\% yield) ${ }^{1} \mathrm{H}$ NMR $\left(400 \mathrm{MHz}, \mathrm{CDCl}_{3}\right) \delta 8.49(\mathrm{~s}, 2 \mathrm{H}), 7.62(\mathrm{~d}, \mathrm{~J}=8.52$ $\mathrm{Hz}, 2 \mathrm{H}), 7.51(\mathrm{~d}, \mathrm{~J}=8.56 \mathrm{~Hz}, 2 \mathrm{H}), 7.36-7.32(\mathrm{~m}, 3 \mathrm{H}), 7.01(\mathrm{sext}, \mathrm{J}=4.56,3.6,0.8 \mathrm{~Hz}, 1 \mathrm{H}), 6.88$ $(\mathrm{d}, \mathrm{J}=4.12 \mathrm{~Hz}, 2 \mathrm{H}), 6.85(\mathrm{~d}, \mathrm{~J}=8 \mathrm{~Hz}, 2 \mathrm{H}), 7.62(\mathrm{~d}, \mathrm{~J}=8.52 \mathrm{~Hz}, 2 \mathrm{H}), 7.51(\mathrm{~d}, \mathrm{~J}=8.56 \mathrm{~Hz}, 2 \mathrm{H})$, 7.36-7.32 (m, 3H), $7.01(\mathrm{sext}, \mathrm{J}=4.56,3.6,0.8 \mathrm{~Hz}, 1 \mathrm{H}), 6.88(\mathrm{~d}, \mathrm{~J}=8.52 \mathrm{~Hz}, 2 \mathrm{H}), 6.85(\mathrm{~d}, \mathrm{~J}=8$ Hz, 2H), 3.84 (s, 3H), 3.26 (broad s, 4H), 3.09 (broad s, 4H), 2.88 (broad s, 2H), 2.45 (broad s, 2H), 1.82 (s, 4H); MS (LC/MS, M+H'): 450.70.

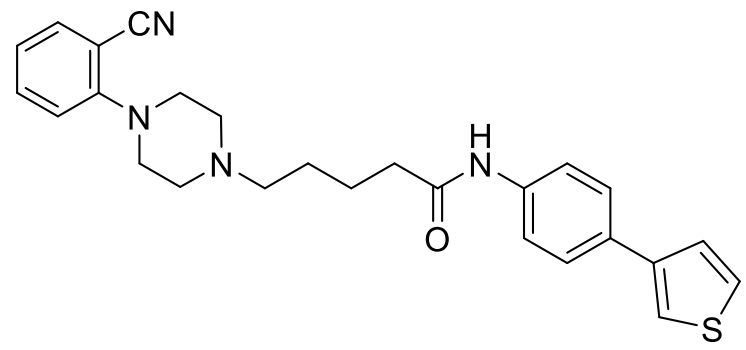

Preparation of 5-(4-(2-cyanophenyl)piperazin-1-yl)-N-(4-(thiophen-3-yl)phenyl)pentanamide (7b): The title compound was prepared according to the procedure for 5-(4- $(3,5-$ dichlorophenyl)piperazin-1-yl)-N-(4-(thiophen-3-yl)phenyl)pentanamide, $\quad$ except $\quad$ 1-(2cyanophenyl)piperazine was substituted for 1-(3,5-dichlorophenyl)piperazine. The product was first purified by normal phase chromatography (10\% methanol in dichloromethane) and was then further purified by reverse phase chromatography (30\% acetonitrile/ $0.1 \%$ formic acid in water) to provide 5-(4-(2-cyanophenyl)piperazin-1-yl)-N-(4-(thiophen-3-yl)phenyl)pentanamide (53\% yield). ${ }^{1} \mathrm{H}$ NMR (400 MHz, $\left.\mathrm{CDCl}_{3}\right) \delta$ 7.58-7.42 (m, 6H), 7.31-7.28 (m, 4H), 7.03-6.95 (m, 2H), 
$3.40($ broad s, 4H), $3.14($ broad s, 4H), 2.89 (broad s, 2H), $2.41($ broad s, 2H), 1.79 (broad s, 4H); MS (LC/MS, M+H'): 445.70.

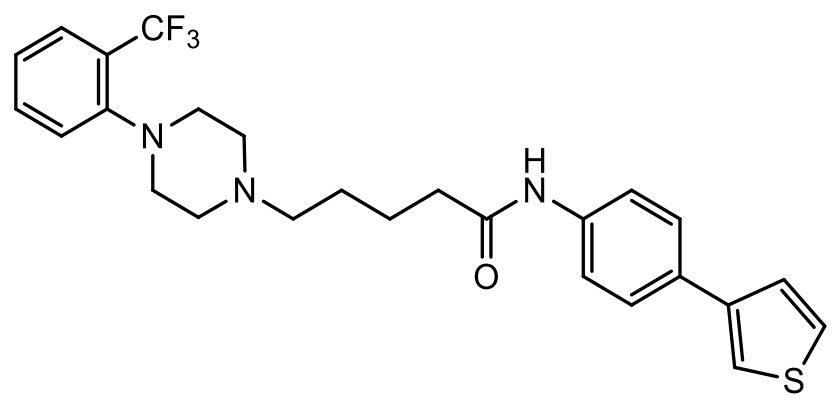

Preparation of N-(4-(thiophen-3-yl)phenyl)-5-(4-(2-(trifluoromethyl)phenyl)piperazin-1yl)pentanamide (7c): The title compound was prepared according to the procedure for 5-(4-(3,5dichlorophenyl)piperazin-1-yl)-N-(4-(thiophen-3-yl)phenyl)pentanamide, $\quad$ except $\quad 1-(2-$ (trifluoromethyl)phenyl)piperazine was substituted for 1-(3,5-dichlorophenyl)piperazine. The product was first purified by normal phase chromatography (10\% methanol in dichloromethane) and was then further purified by reverse phase chromatography (100\% acetonitrile/0.1\% ammonium hydroxide) to provide N-(4-(thiophen-3-yl)phenyl)-5-(4-(2(trifluoromethyl)phenyl)piperazin-1-yl)pentanamide (27\% yield) ${ }^{1} \mathrm{H} \mathrm{NMR}\left(400 \mathrm{MHz}, \mathrm{CDCl}_{3}\right) \delta$ $7.54(\mathrm{dd}, \mathrm{J}=1,0.92 \mathrm{~Hz}, 1 \mathrm{H}), 7.47(\operatorname{broad} \mathrm{s}, 5 \mathrm{H}), 7.41(\mathrm{t}, \mathrm{J}=7.4 \mathrm{~Hz}, 1 \mathrm{H}), 7.32-7.26(\mathrm{~m}, 4 \mathrm{H})$, 7.13(t, J = 7.6 Hz, 1H), $2.88(\mathrm{t}, \mathrm{J}=4.68 \mathrm{~Hz}, 4 \mathrm{H}), 2.52(\operatorname{broad} \mathrm{s}, 4 \mathrm{H}), 2.34(\operatorname{sext}, \mathrm{J}=7.88,7.6$, 7.52. 7.28 Hz, 4H), 1.72 (pent, $\mathrm{J}=7.52 \mathrm{~Hz}, 2 \mathrm{H}), 1.55$ (pent, $\mathrm{J}=7 \mathrm{~Hz}, 2 \mathrm{H}$ ); $\mathrm{MS}\left(\mathrm{LC} / \mathrm{MS}, \mathrm{M}+\mathrm{H}^{+}\right.$): 488.65 .<smiles>N#Cc1cccc(N2CCN(CCCCC(=O)Nc3ccc(-c4ccsc4)cc3)CC2)c1</smiles> 
Preparation of 5-(4-(3-cyanophenyl)piperazin-1-yl)-N-(4-(thiophen-3-yl)phenyl)pentanamide (7d): The title compound was prepared according to the procedure for 5-(4- $(3,5-$ dichlorophenyl)piperazin-1-yl)-N-(4-(thiophen-3-yl)phenyl)pentanamide, $\quad$ except $\quad 1-(3-$ cyanophenyl)piperazine was substituted for 1-(3,5-dichlorophenyl)piperazine. The product was first purified by normal phase chromatography (10\% methanol in dichloromethane) and was then further purified by reverse phase chromatography (85\% acetonitrile/ $0.1 \%$ ammonium hydroxide in water) to provide 5-(4-(3-cyanophenyl)piperazin-1-yl)-N-(4-(thiophen-3yl)phenyl)pentanamide (34\% yield). ${ }^{1} \mathrm{H} \mathrm{NMR}\left(400 \mathrm{MHz}, \mathrm{CDCl}_{3}\right) \delta 7.49$ (s, 4H), 7.33-7.28 (m, 4H), 7.25-7.21 (m, 1H), 7.03-7.00 (m, 3H), $3.16(\mathrm{t}, \mathrm{J}=5 \mathrm{~Hz}, 4 \mathrm{H}), 2.55(\mathrm{t}, \mathrm{J}=4.76 \mathrm{~Hz}, 4 \mathrm{H})$, 2.42-2.33 (m, 4H), 1.72 (pent, $\mathrm{J}=7.44 \mathrm{~Hz}, 2 \mathrm{H}$ ), 1.57 (pent, $\mathrm{J}=6.92 \mathrm{~Hz}, 2 \mathrm{H}$ ); MS (LC/MS, $\left.\mathrm{M}+\mathrm{H}^{+}\right): 445.70$.

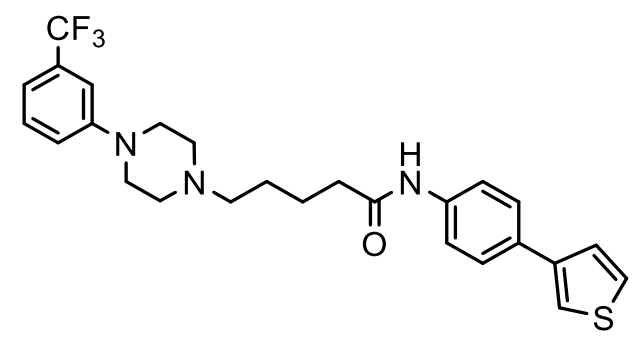

Preparation of N-(4-(thiophen-3-yl)phenyl)-5-(4-(3-(trifluoromethyl)phenyl)piperazin-1yl)pentanamide (7e): The title compound was prepared according to the procedure for 5-(4-(3,5dichlorophenyl)piperazin-1-yl)-N-(4-(thiophen-3-yl)phenyl)pentanamide, $\quad$ except $\quad$ 1-(3(trifluoromethyl)phenyl)piperazine was substituted for 1-(3,5-dichlorophenyl)piperazine. The product was purified by normal phase chromatography (100\% ethyl acetate to $10 \%$ methanol in dichloromethane) to provide N-(4-(thiophen-3-yl)phenyl)-5-(4-(3(trifluoromethyl)phenyl)piperazin-1-yl)pentanamide (45\% yield). ${ }^{1} \mathrm{H}$ NMR (400 $\left.\mathrm{MHz}, \mathrm{CDCl}_{3}\right) \delta$ $7.48(\mathrm{~s}, 4 \mathrm{H}), 7.33-7.28(\mathrm{~m}, 4 \mathrm{H}), 7.26(\mathrm{~s}, 1 \mathrm{H}), 7.02-6.95(\mathrm{~m}, 3 \mathrm{H}), 3.17(\mathrm{t}, \mathrm{J}=5.08 \mathrm{~Hz}, 4 \mathrm{H}), 2.54$ 
$(\mathrm{t}, \mathrm{J}=6.12 \mathrm{~Hz}, 4 \mathrm{H}), 2.36(\mathrm{~m}, 4 \mathrm{H}), 1.73$ (pent, $\mathrm{J}=7.48 \mathrm{~Hz}, 2 \mathrm{H}), 1.57$ (pent, $\mathrm{J}=7.04 \mathrm{~Hz}, 2 \mathrm{H}$ ); MS $\left(\mathrm{LC} / \mathrm{MS}, \mathrm{M}+\mathrm{H}^{+}\right): 488.60$.<smiles>O=C(CCCCN1CCN(c2cccc(Cl)c2Cl)CC1)Nc1ccc(-c2ccsc2)cc1</smiles>

Preparation of 5-(4-(2,3-dichlorophenyl)piperazin-1-yl)-N-(4-(thiophen-3yl)phenyl)pentanamide (7f): The title compound was prepared according to the procedure for 5(4-(3,5-dichlorophenyl)piperazin-1-yl)-N-(4-(thiophen-3-yl)-phenyl)pentanamide, except 1-(2,3dichlorophenyl)piperazine was substituted for 1-(3,5-dichlorophenyl)piperazine. The product was purified by normal phase chromatography (100\% ethyl acetate) to provide 5-(4-(2,3dichlorophenyl)piperazin-1-yl)-N-(4-(thiophen-3-yl)phenyl)pentanamide (65\% yield) ${ }^{1} \mathrm{H}$ NMR $\left(400 \mathrm{MHz}, \mathrm{CDCl}_{3}\right) \delta 7.48(\mathrm{~s}, 4 \mathrm{H}), 7.33-7.27(\mathrm{~m}, 3 \mathrm{H}), 7.10-7.04(\mathrm{~m}, 3 \mathrm{H}), 6.87(\mathrm{dd}, \mathrm{J}=4.92,2.32$ Hz, 1H), 3.02 (s, 4H), $2.63(\mathrm{~s}, 4 \mathrm{H}), 2.45(\mathrm{t}, \mathrm{J}=7.44 \mathrm{~Hz}, 2 \mathrm{H}), 2.34(\mathrm{t}, \mathrm{J}=7.4 \mathrm{~Hz}, 2 \mathrm{H}), 1.74$ (pent, $\mathrm{J}=7.68 \mathrm{~Hz}, 2 \mathrm{H}), 1.58$ (pent, $\mathrm{J}=8.04 \mathrm{~Hz}, 2 \mathrm{H}) ; \mathrm{MS}\left(\mathrm{LC} / \mathrm{MS}, \mathrm{M}+\mathrm{H}^{+}\right): 488.60$.

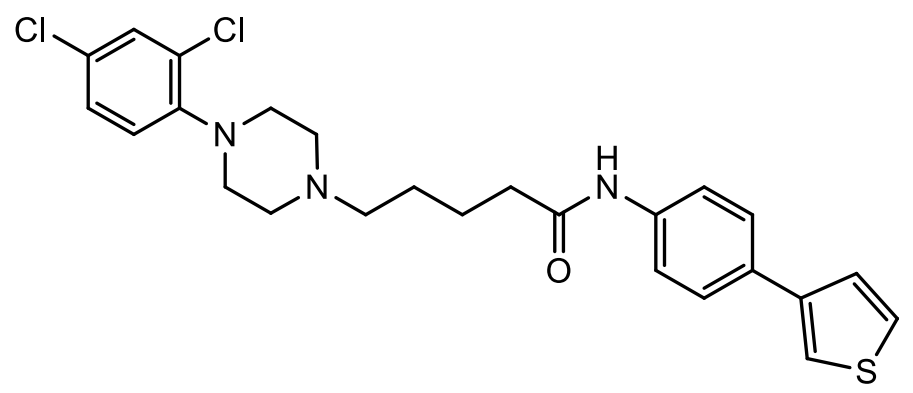

Preparation of 5-(4-(2,4-dichlorophenyl)piperazin-1-yl)-N-(4-(thiophen-3-yl)phenyl) pentanamide (7g): The title compound was prepared according to the procedure for 5-(4-(3,5dichlorophenyl)piperazin-1-yl)-N-(4-(thiophen-3-yl)-phenyl)pentanamide, $\quad$ except $\quad$ 1-(2,4- 
dichlorophenyl)piperazine was substituted for 1-(3,5-dichlorophenyl)piperazine. The product was purified by normal phase chromatography (100\% ethyl acetate) to provide 5-(4-(2,4dichlorophenyl)piperazin-1-yl)-N-(4-(thiophen-3-yl)phenyl)pentanamide (42\% yield). ${ }^{1} \mathrm{H}$ NMR $\left(400 \mathrm{MHz}, \mathrm{CDCl}_{3}\right) \delta 7.59(\mathrm{~s}, 1 \mathrm{H}), 7.52-7.46(\mathrm{~m}, 4 \mathrm{H}), 7.33-7.28(\mathrm{~m}, 4 \mathrm{H}), 7.11(\mathrm{dd}, \mathrm{J}=6.16,2.44$ Hz, 1H), $6.89(\mathrm{~d}, \mathrm{~J}=8.64 \mathrm{~Hz}, 1 \mathrm{H}), 3.02(\mathrm{~s}, 4 \mathrm{H}), 2.64(\mathrm{~s}, 4 \mathrm{H}), 2.45(\mathrm{t}, \mathrm{J}=7.2 \mathrm{~Hz}, 2 \mathrm{H}), 2.36(\mathrm{t}, \mathrm{J}=$ $7.24 \mathrm{~Hz}, 2 \mathrm{H}), 1.74(\mathrm{~m}, 2 \mathrm{H}), 1.61(\mathrm{~m}, 2 \mathrm{H}) ; \mathrm{MS}\left(\mathrm{LC} / \mathrm{MS}, \mathrm{M}+\mathrm{H}^{+}\right): 488.60$.

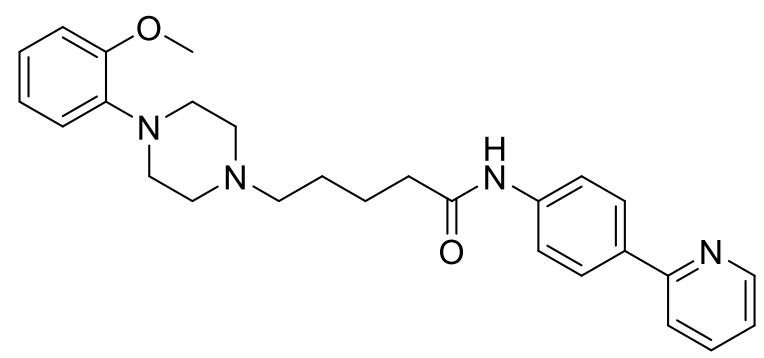

5-(4-(2-methoxyphenyl)piperazin-1-yl)-N-(4-(pyridin-2-yl)phenyl)pentanamide (7i): The title compound was prepared according to the procedure for 5-(4-(2,3-dichlorophenyl)piperazin-1yl)-N-(4-(pyridin-2-yl)phenyl)pentanamide, except 1-(2-methoxyphenyl)piperazine was substituted for 1-(2,3-dichlorophenyl)piperazine (71\% yield). ${ }^{1} \mathrm{H}$ NMR (400 MHz, DMSO) $\delta$ $8.62(\mathrm{dd}, 1 \mathrm{H}, \mathrm{J}=7.4,2.2 \mathrm{~Hz}), 8.05(\mathrm{~d}, 2 \mathrm{H}, \mathrm{J}=7.7 \mathrm{~Hz}), 7.91(\mathrm{~d}, 1 \mathrm{H}, \mathrm{J}=7.1 \mathrm{~Hz}), 7.83(\mathrm{~m}, 1 \mathrm{H}, \mathrm{J}=$ 6.1, $2.1 \mathrm{~Hz}), 7.22(\mathrm{~d}, 2 \mathrm{H}, \mathrm{J}=7.4 \mathrm{~Hz}), 7.29(\mathrm{~m}, 1 \mathrm{H}), 6.91(\mathrm{~m}, 2 \mathrm{H}), 6.87(\mathrm{~m}, 2 \mathrm{H}), 3.82(\mathrm{~s}, 3 \mathrm{H})$, $3.38(\mathrm{~m}, 8 \mathrm{H}), 2.98(\mathrm{~m}, 2 \mathrm{H}), 2.45(\mathrm{~m}, 2 \mathrm{H}), 1.67(\mathrm{~m}, 2 \mathrm{H}), 1.52(\mathrm{~m}, 2 \mathrm{H})$. MS (LC/MS, M+H $\left.\mathrm{H}^{+}\right)$: 445.

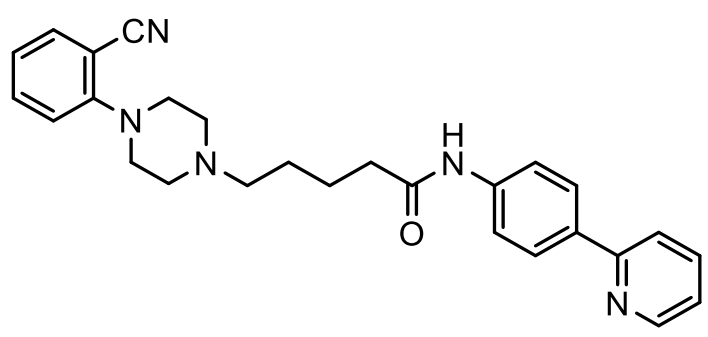


Preparation of 5-(4-(2-cyanophenyl)piperazin-1-yl)-N-(4-(pyridin-2-yl)phenyl)pentanamide (7j): The title compound was prepared according to the procedure for 5-(4-(2,3dichlorophenyl)piperazin-1-yl)-N-(4-(pyridin-2-yl)phenyl)pentanamide, except 2-(piperazin-1yl)benzonitrile was substituted for 1-(2,3-dichlorophenyl)piperazine. The reaction mixture was purified by normal phase chromatography (10\% methanol/0.1\% ammonium hydroxide in dichloromethane) and normal amine phase (RediSep Rf Gold $\mathbb{R}$ Amine \#: 69-2203-507) chromatography (100\% hexane to $100 \%$ ethyl acetate) to provide 5-(4-(2cyanophenyl)piperazin-1-yl)-N-(4-(pyridin-2-yl)phenyl)pentanamide (41\% yield): ${ }^{1} \mathrm{H}$ NMR (400 $\left.\mathrm{MHz}, \mathrm{CDCl}_{3}\right) \delta$ 8.60-8.59 (d, J = 4.64 Hz, 1H), 7.92-7.89 (d, J = 8.64 Hz, 2H), 7.67-7.62 (m, 2H), 7.60-7.58 (d, J = 8.44 Hz, 2H), 7.50-7.48 (dd, J = 6.56, 1.56, $1.4 \mathrm{~Hz}, 1 \mathrm{H}), 7.46($ broad s, $1 \mathrm{H})$, 7.43-7.38 (td, J = 6.64, 6.48, 1.56, $1.08 \mathrm{~Hz}, 1 \mathrm{H}), 7.15-7.12(\mathrm{~m}, 1 \mathrm{H}), 6.93-6.92(\mathrm{~d}, \mathrm{~J}=7.92 \mathrm{~Hz}$, 2H), 3.19-3.17 (t, J = 4.76, 4.52 Hz, 4H), $4.63($ broad s, 4H), 2.45-2.42 (t, J = 7.44, 7.24 Hz, 2H), 2.38-2.34 (t, $\mathrm{J}=7.48,7.28 \mathrm{~Hz}, 2 \mathrm{H}), 1.78-1.70$ (quint, $\mathrm{J}=7.52,7.48,7.36 \mathrm{~Hz}, 2 \mathrm{H}$ ), 1.61-1.54 (quint, $\mathrm{J}=8.08,7.52,7.28,6.88 \mathrm{~Hz}, 2 \mathrm{H})$; MS (LC/MS, M+H'): 440.80 .

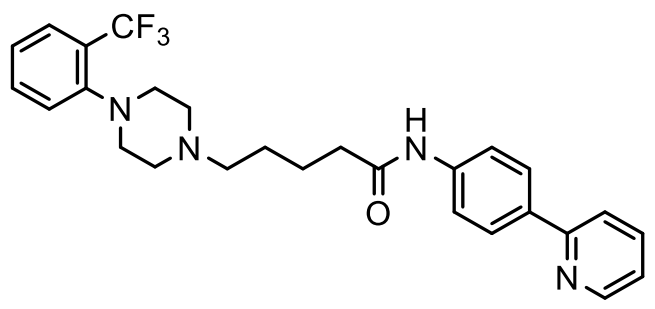

Preparation of N-(4-(pyridin-2-yl)phenyl)-5-(4-(2-(trifluoromethyl)phenyl)piperazin -1-yl)pentanamide (7k): The title compound was prepared according to the procedure for 5-(4(2,3-dichlorophenyl)piperazin-1-yl)-N-(4-(pyridin-2-yl)phenyl)pentanamide, $\quad$ except $\quad$ 1-(2(trifluoromethyl)phenyl)piperazine was substituted for 1-(2,3-dichlorophenyl)piperazine. The reaction mixture was purified by normal phase chromatography (10\% methanol/0.1\% 
ammonium hydroxide in dichloromethane) to give an impure product. The impure product was further purified by normal amine phase (RediSep Rf Gold $\mathbb{R}$ Amine \#: 69-2203-507) chromatography (100\% hexane to $100 \%$ ethyl acetate) to provide N-(4-(pyridin-2-yl)phenyl)-5(4-(2-(trifluoromethyl)phenyl)piperazin-1-yl)pentanamide (32\% yield): ${ }^{1} \mathrm{H}$ NMR (400 MHz, $\left.\mathrm{CDCl}_{3}\right) \delta 8.59(\mathrm{~d}, \mathrm{~J}=4.64 \mathrm{~Hz}, 1 \mathrm{H}), 7.90(\mathrm{~d}, \mathrm{~J}=8.68 \mathrm{~Hz}, 2 \mathrm{H})$, 7.68-7.64 $(\mathrm{m}, 2 \mathrm{H}), 7.62-7.58(\mathrm{~m}$, 2H), $7.54(\mathrm{~d}, \mathrm{~J}=7.8 \mathrm{~Hz}, 1 \mathrm{H}), 7.43(\mathrm{t}, \mathrm{J}=7.76,7.56 \mathrm{~Hz}, 1 \mathrm{H}), 7.29(\mathrm{~d}, \mathrm{~J}=8 \mathrm{~Hz}, 1 \mathrm{H}), 7.16-7.11$ (m, 2H), 2.92 (broad s, 4H), 2.59 (broad s, 4H), 2.39 (broad s, 2H), $2.37(\mathrm{t}, \mathrm{J}=7.08, \mathrm{~Hz}, 2 \mathrm{H})$, 1.75 (broad s, 2H), $1.60($ broad s, 2H); MS (LC/MS, M+H'): 483.8 .

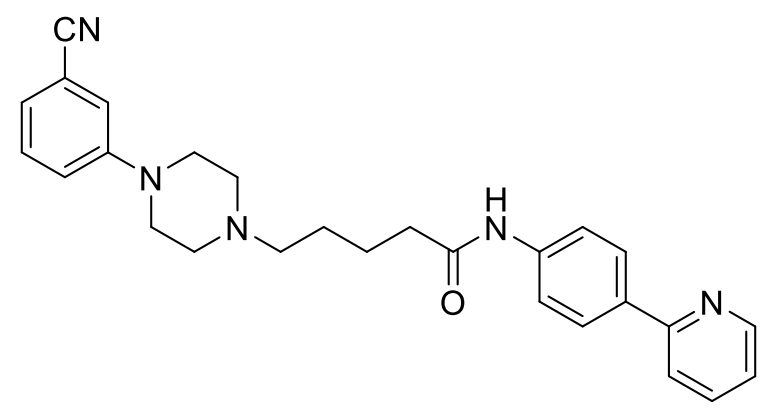

Preparation of N-(4-(pyridin-2-yl)phenyl)-5-(4-(3-cyanophenyl)piperazin-1-yl)pentanamide (7l): The title compound was prepared according to the procedure for 5-(4-(2,3dichlorophenyl)piperazin-1-yl)-N-(4-(pyridin-2-yl)phenyl)pentanamide, $\quad$ except $\quad$ 1-(3cyanophenyl)piperazine was substituted for 1-(2,3-dichlorophenyl)piperazine (37\% yield). ${ }^{1} \mathrm{H}$ NMR (400 MHz, DMSO) $\delta 8.71(\mathrm{~d}, 1 \mathrm{H}, \mathrm{J}=5.4 \mathrm{~Hz}), 8.11(\mathrm{~d}, 2 \mathrm{H}, \mathrm{J}=7.5 \mathrm{~Hz}), 7.97(\mathrm{~d}, 1 \mathrm{H}, \mathrm{J}=$ $7.6 \mathrm{~Hz}), 7.91(\mathrm{dt}, 1 \mathrm{H}, \mathrm{J}=6.9,1.8 \mathrm{~Hz}), 7.78(\mathrm{~d}, 2 \mathrm{H}, \mathrm{J}=7.1 \mathrm{~Hz}), 7.47(\mathrm{~m}, 2 \mathrm{H}), 7.36(\mathrm{dt}, 1 \mathrm{H}, \mathrm{J}=$ 6.1, $1.5 \mathrm{~Hz}), 7.24(\mathrm{~m}, 1 \mathrm{H}), 3.41(\mathrm{bs}, 8 \mathrm{H}), 3.35(\mathrm{~m}, 2 \mathrm{H}), 2.46(\mathrm{~m}, 2 \mathrm{H} 0,1.64(\mathrm{~m}, 4 \mathrm{H}) . \mathrm{MS}$ $\left(\mathrm{LC} / \mathrm{MS}, \mathrm{M}+\mathrm{H}^{+}\right): 440$. 


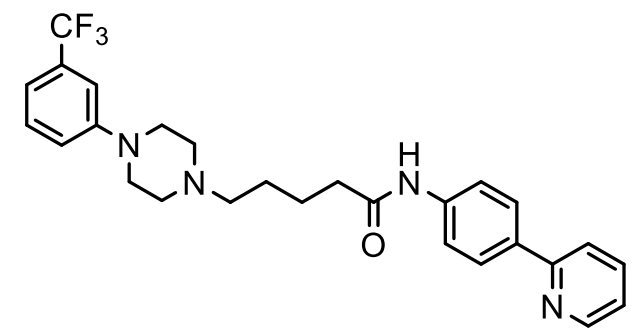

Preparation of N-(4-(pyridin-2-yl)phenyl)-5-(4-(3-(trifluoromethyl)phenyl)piperazin-1yl)pentanamide (7m): The title compound was prepared according to the procedure for 5-(4-(2,3dichlorophenyl)piperazin-1-yl)-N-(4-(pyridin-2-yl)phenyl)pentanamide, except 1-(3(trifluoromethyl)phenyl)piperazine was substituted for 1-(2,3-dichlorophenyl)piperazine. The reaction mixture was purified by normal phase chromatography (10\% methanol/0.1\% ammonium hydroxide in dichloromethane) to give an impure product. The impure product was further purified by normal amine phase (RediSep Rf Gold $\mathbb{R}$ Amine \#: 69-2203-507) chromatography (100\% hexane to $100 \%$ ethyl acetate) to provide $\mathrm{N}$-(4-(pyridin-2-yl)phenyl)-5(4-(3-(trifluoromethyl)phenyl)piperazin-1-yl)pentanamide (43\% yield): ${ }^{1} \mathrm{H}$ NMR (400 MHz, $\left.\mathrm{CDCl}_{3}\right) \delta 8.58(\mathrm{dd}, \mathrm{J}=1.08,0.92 \mathrm{~Hz}, 1 \mathrm{H}), 7.89(\mathrm{~d}, \mathrm{~J}=8.68 \mathrm{~Hz}, 2 \mathrm{H}), 7.68-7.62(\mathrm{~m}, 3 \mathrm{H}), 7.61-$ $7.58(\mathrm{~d}, \mathrm{~J}=8.56 \mathrm{~Hz}, 2 \mathrm{H}), 7.49-7.47(\mathrm{~d}, \mathrm{~J}=6.36 \mathrm{~Hz}, 1 \mathrm{H}), 7.42-7.38(\mathrm{dt}, \mathrm{J}=6.6,6.28,1.64,1.24$ $\mathrm{Hz}, 1 \mathrm{H}), 7.19-7.11(\mathrm{~m}, 1 \mathrm{H}), 6.92(\mathrm{t}, \mathrm{J}=7.84,7.56 \mathrm{~Hz}, 2 \mathrm{H}), 3.18(\mathrm{t}, \mathrm{J}=4.8,4.6 \mathrm{~Hz}, 4 \mathrm{H}), 2.62(\mathrm{t}$, $\mathrm{J}=4.52,4.4 \mathrm{~Hz}, 4 \mathrm{H}), 2.43(\mathrm{t}, \mathrm{J}=7.44,7.28 \mathrm{~Hz}, 2 \mathrm{H}), 2.35(\mathrm{t}, \mathrm{J}=7.48,7.32 \mathrm{~Hz}, 2 \mathrm{H}), 1.76-1.69$ (quint, 2H), 1.60-1.53 (quint, 2H); MS (LC/MS, M+H'): 483.

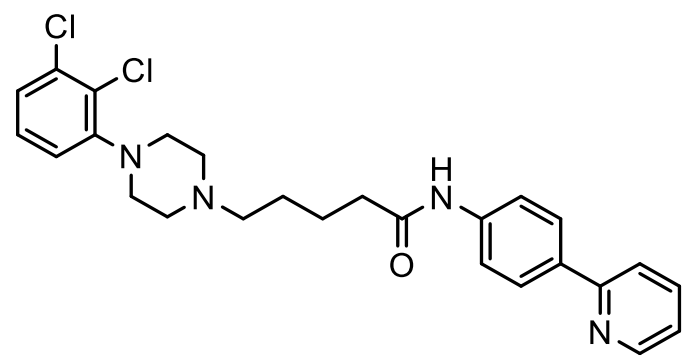


Preparation of 5-(4-(2,3-dichlorophenyl)piperazin-1-yl)-N-(4-(pyridin-2-yl)phenyl)pentanamide (7n): A solution of 5-bromo-N-(4-(pyridin-2-yl)phenyl)pentanamide $(0.191 \mathrm{~g}, 0.573 \mathrm{mmol}), 1$ (2,3-dichlorophenyl)piperazine $(0.11 \mathrm{ml}, 0.573 \mathrm{mmol})$ and potassium carbonate $(0.24 \mathrm{~g}, 1.72$ $\mathrm{mmol}, 3.0 \mathrm{eq})$ in acetone $(6 \mathrm{ml})$ was refluxed at $56^{\circ} \mathrm{C}$ for 24 hours. After 24 hours, the reaction was cooled and filtered to give a filtrate. The filtrate was purified by normal phase chromatography (100\% dichloromethane to $100 \%$ ethyl acetate to $10 \%$ methanol in dichloromethane) to give an impure product. The impure product was further purified by normal amine phase (RediSep Rf Gold $\mathbb{R}$ Amine \#: 69-2203-507) chromatography (100\% hexane to $100 \%$ ethyl acetate) to provide 5-(4-(2,3-dichlorophenyl)piperazin-1-yl)-N-(4-(pyridin-2yl)phenyl)pentanamide (104 mg, 36\% yield): ${ }^{1} \mathrm{H} \mathrm{NMR}\left(400 \mathrm{MHz}, \mathrm{CDCl}_{3}\right) \delta 8.58$ (dd, J = 3.24, 1.2, $0.6 \mathrm{~Hz}, 1 \mathrm{H}), 7.89(\mathrm{~d}, \mathrm{~J}=8.68 \mathrm{~Hz}, 2 \mathrm{H}), 7.68-7.62(\mathrm{~m}, 3 \mathrm{H}), 7.61-7.58(\mathrm{~m}, 2 \mathrm{H}), 7.19-7.11(\mathrm{~m}$, 1H), 7.10-7.04 (m, 2H), 6.88-6.85 (dd, J = 4.88, 2.36, $2.32 \mathrm{~Hz}, 1 \mathrm{H}), 3.02$ (broad s, 4H), 2.61 (broad s, 4H), $2.43(\mathrm{t}, \mathrm{J}=7.44,7.24 \mathrm{~Hz}, 2 \mathrm{H}), 2.36(\mathrm{t}, \mathrm{J}=7.48,7.28 \mathrm{~Hz}, 2 \mathrm{H}$ ), 1.77-1.70 (quint, 2H), 1.61-1.54 (quint, 2H); MS (LC/MS, M+H+1): 484.70.

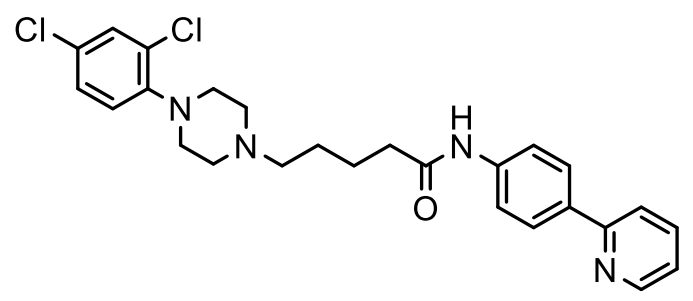

Preparation of 5-(4-(2,4-dichlorophenyl)piperazin-1-yl)-N-(4-(pyridin-2-yl)phenyl)pentanamide (7o): The title compound was prepared according to the procedure for 5-(4-(2,3dichlorophenyl)piperazin-1-yl)-N-(4-(pyridin-2-yl)phenyl)pentanamide, $\quad$ except $\quad 1-(2,4-$ dichlorophenyl)piperazine was substituted for 1-(2,3-dichlorophenyl)piperazine. The reaction mixture was purified by normal phase chromatography $(10 \%$ methanol/ $/ .1 \%$ ammonium 
hydroxide in dichloromethane) and normal amine phase (RediSep Rf Gold $\mathbb{R}$ Amine \#: 69-2203507) chromatography (100\% hexane to $100 \%$ ethyl acetate) to provide 5-(4-(2,4dichlorophenyl)piperazin-1-yl)-N-(4-(pyridin-2-yl)phenyl)pentanamide ( $31 \%$ yield). ${ }^{1} \mathrm{H}$ NMR $\left(400 \mathrm{MHz}, \mathrm{CDCl}_{3}\right) \delta 8.61-8.59(\mathrm{td}, \mathrm{J}=2.24,1.28,0.96,0.64 \mathrm{~Hz}, 1 \mathrm{H}), 7.90(\mathrm{~d}, \mathrm{~J}=8.68 \mathrm{~Hz}, 2 \mathrm{H})$, 7.69-7.64 (m, 2H), 7.64-7.58 (m, 2H), 7.50 (broad s, 1H), $7.28(\mathrm{~d}, \mathrm{~J}=2.4 \mathrm{~Hz}, 1 \mathrm{H}), 7.15-7.10(\mathrm{~m}$, 2H), $6.88(\mathrm{~d}, \mathrm{~J}=8.64 \mathrm{~Hz}, 1 \mathrm{H}), 3.02($ broad s, 4H), $2.63($ broad s, 4H), $2.46(\mathrm{t}, \mathrm{J}=7.52,7.2 \mathrm{~Hz}$, 2H), 2.38 (t, J = 7.44, 7.28 Hz, 2H), 1.79-1.72 (quint, 2H), 1.64-1.57 (quint, 2H); MS (LC/MS, $\left.\mathrm{M}+\mathrm{H}^{+}\right): 484$.

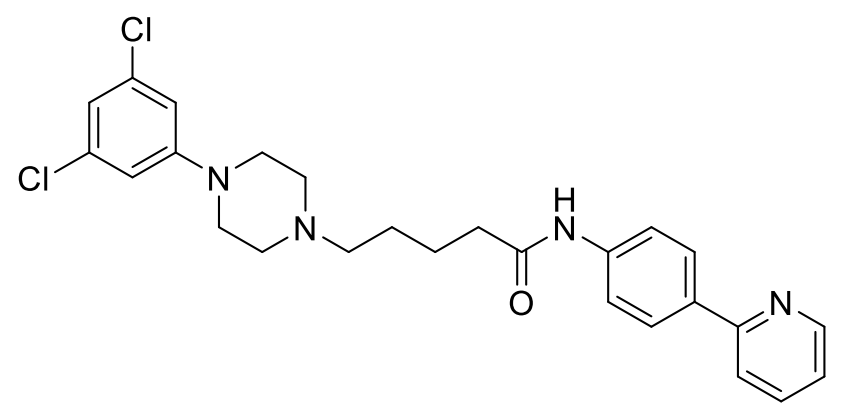

Preparation of 5-(4-(3,5-dichlorophenyl)piperazin-1-yl)-N-(4-(pyridin-2-yl)phenyl)pentanamide (7p): The title compound was prepared according to the procedure for 5-(4-(2,3dichlorophenyl)piperazin-1-yl)-N-(4-(pyridin-2-yl)phenyl)pentanamide, $\quad$ except $\quad$ 1-(2,4dichlorophenyl)piperazine was substituted for 1-(2,3-dichlorophenyl)piperazine (46\% yield) (400 MHz, DMSO) $\delta 8.69(\mathrm{DD}, 1 \mathrm{H}, \mathrm{J}=5.9,1.5 \mathrm{~Hz}), 8.11(\mathrm{~d}, 2 \mathrm{H}, \mathrm{J}=7.2 \mathrm{~Hz}), 7.97(\mathrm{~d}, 1 \mathrm{H}, \mathrm{J}=7.2$ Hz), $7.91(\mathrm{dt}, 1 \mathrm{H}, \mathrm{J}=5.5,1.2 \mathrm{~Hz}), 7.78(\mathrm{~d}, 2 \mathrm{H}, \mathrm{J}=7.1 \mathrm{~Hz}), 7.37(\mathrm{~m}, 1 \mathrm{H}), 6.98(\mathrm{bs}, 2 \mathrm{H}), 6.92(\mathrm{bs}$, 1H), $3.51(\mathrm{~m}, 8 \mathrm{H}), 3.41(\mathrm{~m}, 2 \mathrm{H}), 2.48(\mathrm{~m}, 2 \mathrm{H}), 1.71(\mathrm{~m}, 2 \mathrm{H}), 1.62(\mathrm{~m}, 2 \mathrm{H}) . \mathrm{MS}(\mathrm{LC} / \mathrm{MS}$, $\left.\mathrm{M}+\mathrm{H}^{+}\right): 484$. 


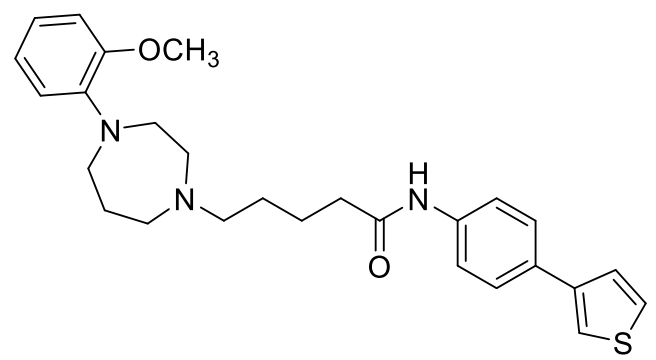

Preparation of 5-(4-(2-methoxyphenyl)-1,4-diazepan-1-yl)-N-(4-(thiophen-3yl)phenyl)pentanamide (7q): The title compound was prepared according to the procedure for 5(4-(3,5-dichlorophenyl)piperazin-1-yl)-N-(4-(thiophen-3-yl)phenyl)pentanamide, except 1-(2methoxyphenyl)-1,4-diazepane was substituted for 1-(3,5-dichlorophenyl)piperazine. The reaction mixture was first purified by normal phase chromatography $(10 \%$ methanol in dichloromethane) to give a partially pure desired product. The impure product was further purified by reverse phase chromatography $(50 \%$ acetonitrile/ $0.1 \%$ formic acid in water) to provide 5-(4-(2-methoxyphenyl)-1,4-diazepan-1-yl)-N-(4-(thiophen-3-yl)phenyl)pentanamide (48\% yield) ${ }^{1} \mathrm{H}$ NMR (400 MHz, $\left.\mathrm{CDCl}_{3}\right) \delta 7.62($ broad s, $2 \mathrm{H}), 7.47$ (broad s, 2H), 7.31-7.29 (m, 3H), 6.89 (broad s, 1H), 6.82-6.78 (m, 3H), 3.74 (s, 3H), 3.33 (broad s, 6H), 3.19 (broad s, 2H), $3.16($ broad s, 2H), $2.44($ broad s, 2H), $2.28($ broad s, 2H), 1.81 (broad s, 2H); MS (LC/MS, $\left.\mathrm{M}+\mathrm{H}^{+}\right): 464$.

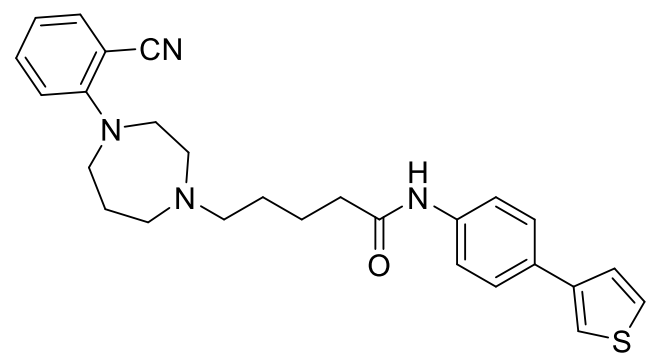

Preparation of 5-(4-(2-cyanophenyl)-1,4-diazepan-1-yl)-N-(4-(thiophen-3yl)phenyl)pentanamide (7r): The title compound was prepared according to the procedure for (4-(3,5-dichlorophenyl)piperazin-1-yl)-N-(4-(thiophen-3-yl)phenyl)pentanamide, except 2-(1,4- 
diazepan-1-yl)benzonitrile was substituted for 1-(2-methoxyphenyl)-1,4-diazepane to provide 5(4-(2-cyanophenyl)-1,4-diazepan-1-yl)-N-(4-(thiophen-3-yl)phenyl)pentanamide (44\% yield). ${ }^{1} \mathrm{H}$ NMR (400 MHz, CDCl 3 ) $\delta 7.69($ broad s, 1H), 7.45-7.43 (d, J = 8.64 Hz, 2H), 7.40-7.38 (d, J = $8.68 \mathrm{~Hz}, 2 \mathrm{H}), 7.35-7.33(\mathrm{dd}, \mathrm{J}=6.12,1.64 \mathrm{~Hz}, 1 \mathrm{H}), 7.25-7.13(\mathrm{~m}, 4 \mathrm{H}), 6.71-6.69(\mathrm{~d}, \mathrm{~J}=8.64$ $\mathrm{Hz}, 1 \mathrm{H}), 6.64-6.61(\mathrm{dt}, \mathrm{J}=7.28,7.2,0.52,0.36 \mathrm{~Hz}, 1 \mathrm{H}), 3.53-3.50(\mathrm{~m}, 2 \mathrm{H}), 3.44-3.41(\mathrm{t}, \mathrm{J}=6$, $5.96 \mathrm{~Hz}, 2 \mathrm{H}), 2.76-2.73(\mathrm{t}, \mathrm{J}=4.56,4.52 \mathrm{~Hz}, 2 \mathrm{H}), 2.59-2.57(\mathrm{t}, \mathrm{J}=5.28,5.2 \mathrm{~Hz}, 2 \mathrm{H}), 2.43-2.39$ (t, J = 7.32, 7.16 Hz, 2H), 2.28-2.23 (t, J = 7.44, 7.32 Hz, 2H), 1.92-1.87 (m, 2H), 1.65-1.58 (quint, $\mathrm{J}=7.56,7.44,7.36,7.28 \mathrm{~Hz}, 2 \mathrm{H}$ ), 1.49-1.42 (quint, $\mathrm{J}=7.88,7.52,7.046 .84 \mathrm{~Hz}, 2 \mathrm{H}$ ); $\mathrm{MS}$ (LC/MS, M+H'): 459 .

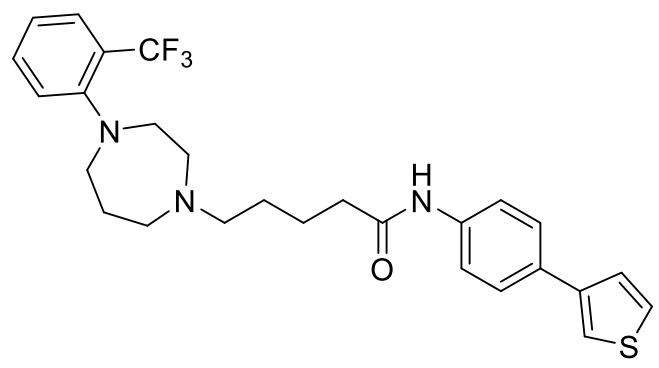

Preparation of N-(4-(thiophen-3-yl)phenyl)-5-(4-(2-(trifluoromethyl)phenyl)-1,4-diazepan-1yl)pentanamide (7s): The title compound was prepared according to the procedure for -(4- $(3,5-$ dichlorophenyl)piperazin-1-yl)-N-(4-(thiophen-3-yl)phenyl)pentanamide, $\quad$ except $\quad$ 1-(2(trifluoromethyl)phenyl)-1,4-diazepane was substituted for 1-(2-methoxyphenyl)-1,4-diazepane to provide N-(4-(thiophen-3-yl)phenyl)-5-(4-(2-(trifluoromethyl)phenyl)-1,4-diazepan-1-yl) -pentanamide (51\% yield). ${ }^{1} \mathrm{H}$ NMR (400 MHz, $\left.\mathrm{CDCl}_{3}\right) \delta$ 7.64-7.56 (m, 6H), 7.52-7.48 (t, J = 7.32, 7.08 Hz, 2H), 7.42-7.34 (m, 4H), 7.21-7.17(t, J = 7.64, 7.56 Hz, 1H), 3.19-3.14 (m, 4H), $2.90-2.87(\mathrm{~d}, \mathrm{~J}=5.88,5.72 \mathrm{~Hz}, 2 \mathrm{H}), 2.83-2.80(\mathrm{~m}, 2 \mathrm{H}), 2.65-2.62(\mathrm{t}, \mathrm{J}=7.32,7.16 \mathrm{~Hz}, 2 \mathrm{H})$, $2.46-2.43(\mathrm{t}, \mathrm{J}=7.48,7.32 \mathrm{~Hz}, 2 \mathrm{H}), 1.96-1.90$ (quint, $\mathrm{J}=6.12,6.08,5.88 \mathrm{~Hz}, 2 \mathrm{H}$ ), $1.86-1.78$ 
(quint, $\mathrm{J}=7.56,7.44,7.28 \mathrm{~Hz}, 2 \mathrm{H}$ ), 1.68-1.61 (quint, $\mathrm{J}=7.8,7.6,6.96,6.92 \mathrm{~Hz}, 2 \mathrm{H}$ ); $\mathrm{MS}$ $\left(\mathrm{LC} / \mathrm{MS}, \mathrm{M}+\mathrm{H}^{+}\right): 502$.

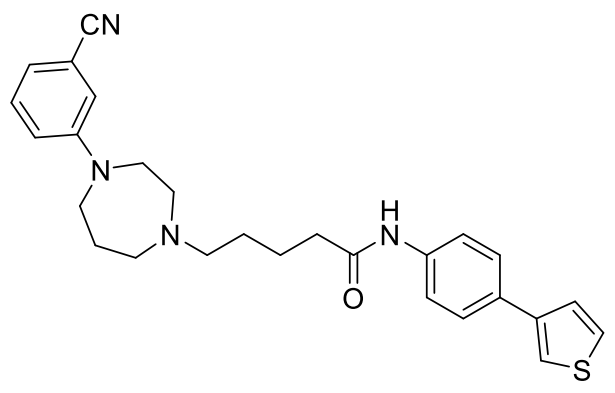

Preparation of 5-(4-(3-cyanophenyl)-1,4-diazepan-1-yl)-N-(4-(thiophen-3yl)phenyl)pentanamide (7t): The title compound was prepared according to the procedure for 5(4-(3,5-dichlorophenyl)piperazin-1-yl)-N-(4-(thiophen-3-yl)phenyl)-pentanamide except 3-(1,4diazepan-1-yl)benzonitrile was substituted for 1-(3,5-dichlorophenyl)piperazine to provide 5-(4(3-cyanophenyl)-1,4-diazepan-1-yl)-N-(4-(thiophen-3-yl)phenyl)pentanamide (39\% yield). ${ }^{1} \mathrm{H}$ $\operatorname{NMR}\left(400 \mathrm{MHz}, \mathrm{CDCl}_{3}\right) \delta$ 7.52-7.46 (m, 4H), 7.33-7.28 (m, 3H), 7.21-7.17 (t, J = 9.08, 7.04 Hz, 2H), 6.88-6.86 (d, J = 7.48 Hz, 1H), 6.79-6.77 (d, J =7.72 Hz, 1H), 3.55 (broad s, 2H), 3.42-3.38 (t, J = 7.72, $3.24 \mathrm{~Hz}, 2 \mathrm{H}), 2.86$ (broad s, 2H), 2.72 (broad s, 2H), 2.62 (broad s, 2H), 2.36-2.32 (t, J = 7, $6.84 \mathrm{~Hz}, 2 \mathrm{H}), 2.04($ broad s, 2H), $1.70($ broad s, 2H), 1.61 (broad s, 2H); MS (LC/MS, $\left.\mathrm{M}+\mathrm{H}^{+}\right): 459$.

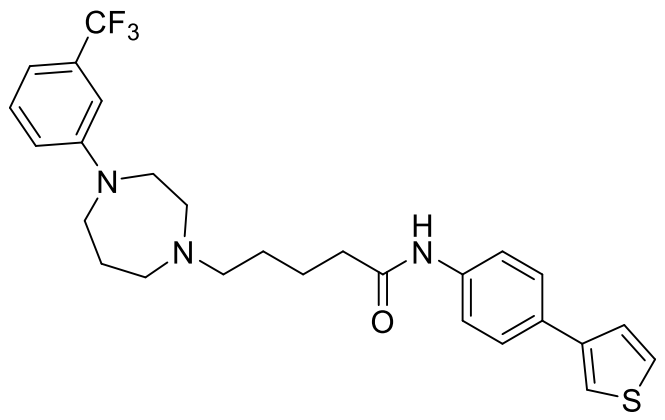

Preparation of N-(4-(thiophen-3-yl)phenyl)-5-(4-(3-(trifluoromethyl)phenyl)-1,4-diazepan-1yl)pentanamide (7u): The title compound was prepared according to the procedure for 5-(4-(3,5- 
dichlorophenyl)piperazin-1-yl)-N-(4-(thiophen-3-yl)phenyl)-pentanamide, $\quad$ except 1-(3(trifluoromethyl)phenyl)-1,4-diazepane was substituted for 1-(3,5-dichlorophenyl)piperazine to provide N-(4-(thiophen-3-yl)phenyl)-5-(4-(3-(trifluoromethyl)phenyl)-1,4-diazepan-1yl)pentanamide (49\% yiled). ${ }^{1} \mathrm{H}$ NMR $\left(400 \mathrm{MHz} \mathrm{CDCl}_{3}\right) \delta$ 7.66-7.64 (d, J = 7.6 Hz, 2H), 7.56$7.54(\mathrm{~d}, \mathrm{~J}=7.76 \mathrm{~Hz}, 2 \mathrm{H}), 7.39-7.28(\mathrm{~m}, 4 \mathrm{H}), 7.00-6.99(\mathrm{~d}, \mathrm{~J}=7.48 \mathrm{~Hz}, 1 \mathrm{H}), 6.86(\mathrm{~s}, 1 \mathrm{H}), 6.82-$ $6.80(\mathrm{~d}, \mathrm{~J}=8.24 \mathrm{~Hz}, 1 \mathrm{H}), 3.76($ broad s, 2H), 3.49 (broad s, 2H), 3.14 (broad s, 2H), 3.03 (broad s, 2H), 2.91 (broad s, 2H), 2.45 (broad s, 2H), 2.29 (broad s, 2H), 1.80 (broad s, 4H); MS $\left(\mathrm{LC} / \mathrm{MS}, \mathrm{M}+\mathrm{H}^{+}\right): 502$.

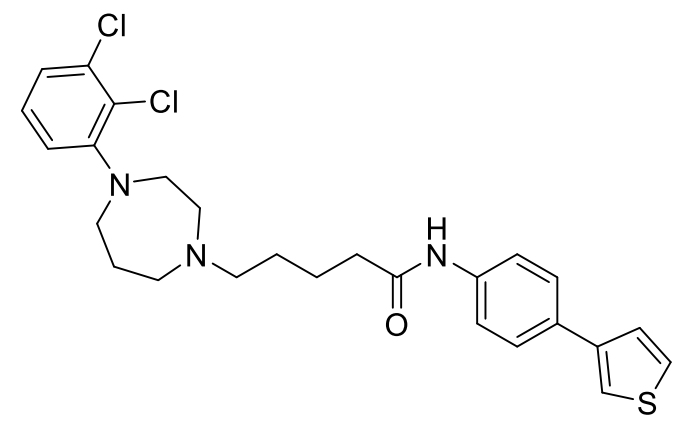

Preparation of 5-(4-(2,3-dichlorophenyl)-1,4-diazepan-1-yl)-N-(4-(thiophen-3yl)phenyl)pentanamide (7v): The title compound was prepared according to the procedure for 5(4-(3,5-dichlorophenyl)piperazin-1-yl)-N-(4-(thiophen-3-yl)phenyl)-pentanamide, except 1-(2,3dichlorophenyl)-1,4-diazepane was substituted for 1-(3,5-dichlorophenyl)piperazine to provide 5-(4-(2,3-dichlorophenyl)-1,4-diazepan-1-yl)-N-(4-(thiophen-3-yl)phenyl)pentanamide $\quad$ (38\% yield). ${ }^{1} \mathrm{H} \mathrm{NMR}\left(400 \mathrm{MHz}, \mathrm{CDCl}_{3}\right) \delta 7.99$ (broad s, 1H), 7.60-7.57 (d, J = 8.6 Hz, 2H), 7.54$7.52(\mathrm{~d}, \mathrm{~J}=8.64 \mathrm{~Hz}, 2 \mathrm{H}), 7.39-7.38(\mathrm{~m}, 1 \mathrm{H}), 7.37-7.34(\mathrm{~m}, 2 \mathrm{H}), 7.09-7.07(\mathrm{t}, \mathrm{J}=3.32,3 \mathrm{~Hz}$ 2H), 6.98-6.95 (dd, J = 3.32, 3.28, 3.08 Hz, 1H), 3.28-3.25 (m, 4H), 2.83-2.78 (m, 4H), 2.58-2.55 $(\mathrm{t}, \mathrm{J}=7.32,7.12, \mathrm{~Hz}, 2 \mathrm{H}), 2.44-2.40(\mathrm{t}, \mathrm{J}=7.48,7.36 \mathrm{~Hz}, 2 \mathrm{H}) ; \mathrm{MS}\left(\mathrm{LC} / \mathrm{MS}, \mathrm{M}+\mathrm{H}^{+}\right): 503$. 


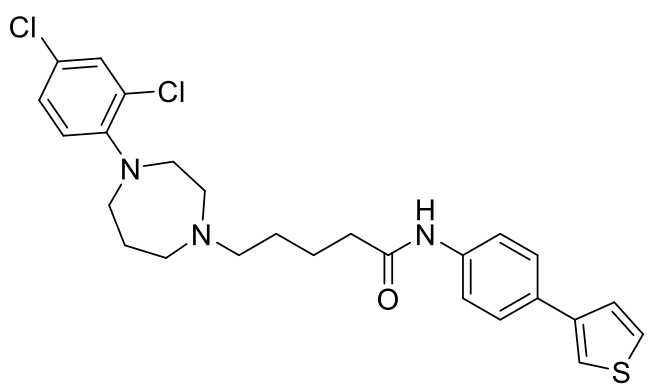

Preparation of 5-(4-(2,4-dichlorophenyl)-1,4-diazepan-1-yl)-N-(4-(thiophen-3yl)phenyl)pentanamide (7w): The title compound was prepared according to the procedure for 5(4-(3,5-dichlorophenyl)piperazin-1-yl)-N-(4-(thiophen-3-yl)phenyl)-pentanamide, except 1-(2,4dichlorophenyl)-1,4-diazepane was substituted for 1-(3,5-dichlorophenyl)piperazine to provide 5-(4-(2,4-dichloro-phenyl)-1,4-diazepan-1-yl)-N-(4-(thiophen-3-yl)phenyl)pentanamide $\quad(22 \%$ yield) ${ }^{1} \mathrm{H}$ NMR $\left(400 \mathrm{MHz}, \mathrm{CDCl}_{3}\right) \delta$ 7.67-7.65 (d, J = 8.12 Hz, 2H), 7.55-7.53 (d, J = 8.12 Hz, 2H), 7.39-7.34 (m, 4H), 7.19-7.16 (dd, J = 6.2, 2.44, 2.4 Hz, 1H), 7.00-6.97 (d, J = 8.68 Hz, 1H), 3.39 (broad s, 6H), 3.22 (broad s, 2H), 3.12 (broad s, 2H), 2.49 (broad s, 2H), 2.26 (broad s, 2H), 1.85 (broad s, 4H); MS (LC/MS, M+H'): 503 .

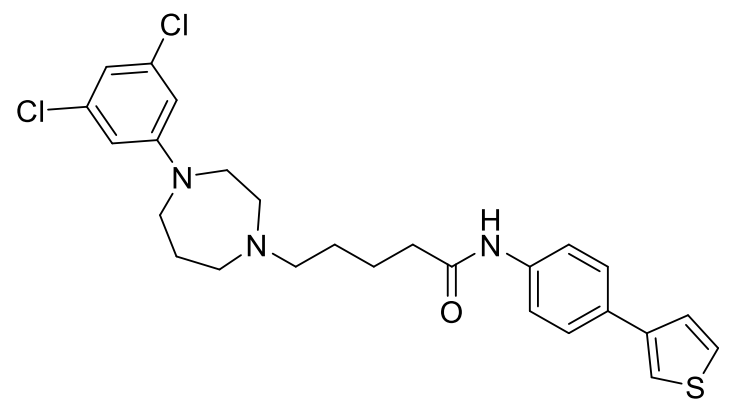

Preparation of 5-(4-(3,5-dichlorophenyl)-1,4-diazepan-1-yl)-N-(4-(thiophen-3yl)phenyl)pentanamide (7x): The title compound was prepared according to the procedure for 5(4-(3,5-dichlorophenyl)piperazin-1-yl)-N-(4-(thiophen-3-yl)phenyl)-pentanamide, except 1-(3,5dichlorophenyl)-1,4-diazepane was substituted for 1-(3,5-dichlorophenyl)piperazine to provide 5-(4-(3,5-dichlorophenyl)-1,4-diazepan-1-yl)-N-(4-(thiophen-3-yl)phenyl)pentanamide $\quad$ (41\% 
yield). ${ }^{1} \mathrm{H}$ NMR $\left(400 \mathrm{MHz}, \mathrm{CDCl}_{3}\right) \delta$ 7.64-7.62 $(\mathrm{d}, \mathrm{J}=8.12 \mathrm{~Hz}, 2 \mathrm{H}), 7.55-7.53(\mathrm{~d}, \mathrm{~J}=8.2 \mathrm{~Hz}$, 2H), 7.40-7.35 (m, 3H), $6.75(\mathrm{~s}, 1 \mathrm{H}), 6.51(\mathrm{~d}, \mathrm{~J}=1.36 \mathrm{~Hz}, 2 \mathrm{H}), 3.73($ broad s, 2H), 3.43-3.40 (d, $\mathrm{J}=5.48,5.4 \mathrm{~Hz}, 2 \mathrm{H}), 3.20($ broad s, 2H), $3.11($ broad s, 2H), $2.99($ broad s, 2H), 2.45 (broad s, 2H), 2.32 (broad s, 2H), 1.80 (broad s, 2H); MS (LC/MS, M+H'): 503.

Computational values: TPSA and cLogP values were calculated using the Dotmatics software suite (Dotmatics LLC The Old Monastery, Windhill Bishops, Stortford Herts, CW23 2ND UK). Competitive radioligand-binding studies. For competitive binding studies, transfected HEK293 cell homogenates were suspended in homogenization buffer and incubated with radioligand $\left[{ }^{125} \mathrm{I}\right] \mathrm{IABN}$, in the presence or absence of inhibitor at $37{ }^{\circ} \mathrm{C}$ for 60 min with $\left[{ }^{125} \mathrm{I}\right] \mathrm{IABN}$ (total volume $=150 \mathrm{ul}$ ) as previously described.Error! Bookmark not defined. Competitive radioligand studies were performed to determine the concentration of inhibitor that inhibits $50 \%$ of the specific binding of the radioligand (IC50 value). The final radioligand concentration was approximately equal to the $\mathrm{Kd}$ value for the binding of the radioligand. For each competition curve, triplicates were performed using two concentrations of inhibitor per decade over five orders of magnitude. Binding was terminated by the addition of cold wash buffer $(10 \mathrm{mM}$ Tris-HCl/150 mM NaCl, $\mathrm{pH}=7.5)$ and filtration over a glass-fiber filter (Pall A/B filters, \#66198). A Packard Cobra Gamma Counter was used to measure the radioactivity of $\left[{ }^{125} \mathrm{I}\right] \mathrm{IABN}$.

The competition curves were modeled for a single binding site using

$$
\text { Bs }=\text { Bo }-((\mathbf{B o}+\mathbf{L}) /(\mathbf{I C 5 0}+\mathbf{L}))
$$

where $\mathrm{Bs}$ is the amount of ligand bound to receptor and Bo is the amount of ligand bound to receptor in the absence of competitive inhibitor. $\mathrm{L}$ is the concentration of the competitive 
inhibitor. The IC50 value is the concentration of competitive inhibitor that inhibits $50 \%$ of the total specific binding. IC50 values were determined using non-linear regression analysis with Table Curve 2D v 5.01 (Jandel, SYSTAT, Systat Software, Inc., San Jose, CA, USA). The values for Bns and Bo were constrained using experimentally derived values. The IC50 values were converted to equilibrium dissociation constants (Ki) using the Cheng and Prusoff (1973) correction. Mean Ki values \pm S.E.M. are reported for at least three independent experiments.

Microsomal Stability Analysis. Test compounds were assessed for microsomal stability by incubating them at $37^{\circ} \mathrm{C}$ in the presence of rat liver microsomes and an NADPH regenerating system.Error! Bookmark not defined. Microsomal protein content was adjusted to give accurate rates of substrate consumption. Analysis was performed by Liquid Chromatography-tandem mass spectrometry (LC/MS/MS) analysis.

Table 1: In vitro pharmacological and ADME screening data for $(\mathbf{7 a})-(7 \mathbf{x})$<smiles>[14CH3]N1CCN(CCCCC(=O)Nc2ccc(Br)cc2)CC1</smiles>

\begin{tabular}{|c|c|c|c|c|c|c|c|c|}
\hline \multirow{2}{*}{ Entry } & \multirow{2}{*}{$\mathrm{Ar}^{1}$} & \multirow{2}{*}{$\mathrm{Ar}^{2}$} & \multirow[t]{2}{*}{$\mathrm{N}$} & $\mathrm{D}_{3}$ & $\mathrm{D}_{2}$ & \multirow{2}{*}{$\mathrm{D}_{2} / \mathrm{D}_{3}$} & \multirow{2}{*}{$\begin{array}{l}\text { Sol } \\
\mu \mathrm{M}\end{array}$} & \multirow{2}{*}{$\frac{\text { RLM }}{\mathrm{T}_{1 / 2} \mathrm{~min}}$} \\
\hline & & & & \multicolumn{2}{|c|}{$\mathrm{K}_{\mathrm{I}}(\mathrm{nM})$} & & & \\
\hline $7 \mathrm{a}$ & 2-OMe-Ph & thiophen-3-yl & 1 & 3.1 & 108 & 34.8 & 7.6 & 5.6 \\
\hline $7 \mathrm{~b}$ & 2-CN-Ph & thiophen-3-yl & 1 & 6.2 & 250 & 40.3 & 8.9 & 6 \\
\hline $7 \mathrm{c}$ & $2-\mathrm{CF}_{3}-\mathrm{Ph}$ & thiophen-3-yl & 1 & 20.5 & 438 & 21.4 & 2 & 19.3 \\
\hline $7 d$ & 3-CN-Ph & thiophen-3-yl & 1 & 14.9 & 8119 & $\begin{array}{l}544.9 \\
\end{array}$ & 2 & 60 \\
\hline $7 \mathrm{e}$ & 3- $-\mathrm{CF}_{3}-\mathrm{Ph}$ & thiophen-3-yl & 1 & 5 & 82.1 & 16.4 & 2 & 60 \\
\hline $7 \mathrm{f}$ & 2,3-Di-Cl-Ph & thiophen-3-yl & 1 & 2 & 216 & 108.0 & 2 & 60 \\
\hline $7 \mathrm{~g}$ & 2,4-Di-Cl-Ph & thiophen-3-yl & 1 & 44.2 & 7500 & 169.7 & 2 & 60 \\
\hline $7 \mathrm{~h}$ & 3,5-Di-Cl-Ph & thiophen-3-yl & 1 & 16.5 & 6482 & 392.8 & 2 & 60 \\
\hline $7 \mathrm{i}$ & 2-OMe-Ph & 2-Pyrdine & 1 & 21.2 & 865 & 40.8 & 184 & 7.7 \\
\hline $7 \mathrm{j}$ & 2-CN-Ph & 2-Pyrdine & 1 & 8 & 715 & 89.4 & 123 & 5.8 \\
\hline $7 \mathrm{k}$ & $2-\mathrm{CF}_{3}-\mathrm{Ph}$ & 2-Pyrdine & 1 & 23.7 & 477.6 & 20.2 & 27 & 3.7 \\
\hline 71 & 3-CN-Ph & 2-Pyrdine & 1 & 11.4 & 1228 & 107.8 & 10.2 & 20.9 \\
\hline
\end{tabular}




\begin{tabular}{|c|l|c|c|c|c|c|c|c|}
\hline $7 \mathrm{~m}$ & 3-CF $-\mathrm{Ph}$ & 2-Pyrdine & 1 & 9.7 & 717.3 & 73.9 & 23.5 & 10.9 \\
\hline $7 \mathrm{n}$ & 2,3-Di-Cl-Ph & 2-Pyrdine & 1 & 22 & 663.7 & 30.2 & 17.2 & 15.1 \\
\hline $7 \mathrm{o}$ & 2,4-Di-Cl-Ph & 2-Pyrdine & 1 & 36 & 10000 & 277.8 & 2.5 & 18.2 \\
\hline $7 \mathrm{p}$ & 3,5-Di-Cl-Ph & 2-Pyrdine & 1 & 4.5 & 353.1 & 78.5 & 2 & 60 \\
\hline $7 \mathrm{q}$ & 2-OMe-Ph & thiophen-3-yl & 2 & 28.6 & 562 & 19.7 & 122 & 2 \\
\hline $7 \mathrm{r}$ & 2-CN-Ph & thiophen-3-yl & 2 & 35.3 & 976 & 27.6 & 31.7 & 8.5 \\
\hline $7 \mathrm{~s}$ & 2-CF $-\mathrm{Ph}$ & thiophen-3-yl & 2 & 265 & 1912 & 7.2 & 35 & $\mathrm{ND}$ \\
\hline $7 \mathrm{t}$ & 3-CN-Ph & thiophen-3-yl & 2 & 74.7 & 2038 & 27.3 & 30.9 & 2 \\
\hline $7 \mathrm{u}$ & 3-CF $-\mathrm{Ph}$ & thiophen-3-yl & 2 & 92.2 & 6182 & 67.0 & 20.4 & 24.1 \\
\hline $7 \mathrm{v}$ & 2,3-Di-Cl-Ph & thiophen-3-yl & 2 & 12.3 & 383 & 31.1 & 6.8 & 2.7 \\
\hline $7 \mathrm{w}$ & 2,4-Di-Cl-Ph & thiophen-3-yl & 2 & 244 & 1486 & 6.1 & 2.7 & 44.5 \\
\hline $7 \mathrm{x}$ & 3,5-Di-Cl-Ph & thiophen-3-yl & 2 & 253 & 8178 & 32.3 & 2 & 43.2 \\
\hline
\end{tabular}

Acknowledgments: The research reported in this publication was supported by the National Institute on Drug Abuse (NIDA)/National Institutes of Health (NIH) under award numbers DA029840-06A1 and DA023957.

Declaration of interests: The authors have no financial interest to declare.

\section{References and notes}

1. Bose J, Hedden SL, Lipari RN, Park-Lee E Key Substance Use and Mental Health Indicators in the United States: Results from the 2017 National Survey on Drug Use and Health, HHS Publication No. SMA 18-5068, NSDUH Series H-53, Rockville, MD: Center for Behavioral Health Statistics and Quality, Substance Abuse and Mental Health Services Administration, https://www.samhsa.gov/data/

2. NIDA report Health Consequences of Drug Misuse Retrieved from https://www.drugabuse.gov/related-topics/health-consequences-drug-misuse 2019, April 2.

3. Balk JH Dopamine signaling in reward-related behaviors. Front. Neural Circuits 2013:7:152:1-16. DOI: 10.3389/fncir.2013.00152 
4. Wise RA, Koob GF The development and maintenance of drug addiction, Neuropsychopharmacology 2014:39:254-262. doi: 10.1038/npp.2013.261

5. Alonso-Matias L, Reyes-Zamorano E, Gonzalez-Olvera JJ, Cognitive functions of subjects with cocaine and crack dependency disorder during early abstinence Rev. Neurol. 2019;68;271-280. DOI: 10.33588/rn.6807.2018119

6. Substance Abuse and Mental Health Services Administration, Drug Abuse Warning Network, 2011: National Estimates of Drug-Related Emergency Department Visits. HHS Publication No. (SMA) 13-4760, DAWN Series D-39. Rockville, MD: Substance Abuse and Mental Health Services Administration, 2013. https://www.samhsa.gov/data/sites/default/files/DAWN2k11ED/DAWN2k11ED/DAWN 2k11ED.pdf

7. (a) Schierenberg A, van Amsterdam J, van den Brink W, Goudriaan AE, Efficacy of contingency management for cocaine dependence treatment: a review of the evidence. Curr Drug Abuse Rev 2012:5:4:320-331. DOI: 10.2174/1874473711205040006 (b) Penberthy JK, Ait-Daoud N, Vaughan M, Fanning T, Review of treatment for cocaine dependence. Curr Drug Abuse Rev 2010:3:1:49-62. DOI: $10.2174 / 1874473711003010049$

8. (a) Oliveto A, Poling J, Mancino MJ, Feldman Z, Cubells JF, Pruzinsky R, Gonsai K, Cargile C, Sofuoglu M, Chopra MP, Gonzalez-Haddad G, Carroll KM, Kosten TR, Randomized, double-blind, placebo-controlled trial of disulfiram for the treatment of cocaine dependence in methadone-stabilized patients. Drug Alcohol Depend, 2011:113:23:184-191. doi: 10.1016/j.drugalcdep.2010.07.022 (b) McCance-Katz EF, Kosten TR, 
Jatlow P. Disulfiram effects on acute cocaine administration Drug Alcohol Depend 1998:52:1:27-39. doi: 10.1016/s0376-8716(98)00050-7.

9. Johnson BA, Ait-Daoud N, Wang XQ, Penberthy JK, Javors MA, Seneviratne C, Liu L, Topiramate for the treatment of cocaine addiction: a randomized clinical trial. JAMA Psychiatry, 2013:70:12:338-1346. DOI: 10.1001/jamapsychiatry.2013.2295

10. Somoza EC, Winship D, Gorodetzky CW, Lewis D, Ciraulo DA, Galloway GP. Segal SD, Sheehan M, Roache JD, Bickel WK, Jasinski D, Watson DW, Miller SR, Somoza P, Winhusen T, A multisite, double-blind, placebo-controlled clinical trial to evaluate the safety and efficacy of vigabatrin for treating cocaine dependence. JAMA Psychiatry, 2013:70:6:630-637. doi: 10.1001/jamapsychiatry.2013.872.

11. Oliveto A, Poling J, Mancino MJ, Feldman Z, Cubells JF, Pruzinsky R, Gonsai K, Cargile C, Sofuoglu M, Chopra MP, Gonzalez-Haddad G, Carroll KM, Kosten TR. Randomized, double-blind, placebo-controlled trial of disulfiram for the treatment of cocaine dependence in methadone-stabilized patients. Drug Alcohol Depend, 2011:113:23:184-191. doi: 10.1016/j.drugalcdep.2010.07.022 (b) McCance-Katz EF, Kosten TR, Jatlow P. Disulfiram effects on acute cocaine administration. Drug Alcohol Depend 1998:52:1:27-39. doi: 10.1016/s0376-8716(98)00050-7.

12. Placenza FM, Fletcher PJ, Vaccarino FJ, Erb S. Effects of central neurokinin-1 receptor antagonism on cocaine- and opiate-induced locomotor activity and self-administration behaviour in rats Pharmacol Biochem Behav 2006:84:1:94-101. DOI: 10.1016/j.pbb.2006.04.011 
13. Baker DA, McFarland K, Lake RW, Shen H, Toda S, Kalivas PW N-Acetyl cysteineinduced blockade of cocaine-induced reinstatement. Ann N Y Acad Sci 2003:1003:349351. DOI: $10.1196 /$ annals.1300.023

14. Harvey-Lewis C, Li Z, Higgins GA, Fletcher PJ, The 5-HT2C receptor agonist lorcaserin reduces cocaine self-administration, reinstatement of cocaine-seeking and cocaine induced locomotor activity. Neuropharmacology. 2016:101:237-245. DOI: 10.1016/j.neuropharm.2015.09.028

15. Roeper J. Dissecting the diversity of midbrain dopamine neurons. Trends Neurosci 2013:36:6:336-342. DOI: 10.1016/j.tins.2013.03.003

16. Jaber M, Robinson SW, Missale C, Caron MG, Dopamine receptors and brain function. Neuropharmacology. 1996:35:11:1503-1519. DOI: 10.1016/s0028-3908(96)00100-1

17. (a) Staley JK, Mash DC, Adaptive increase in D3 dopamine receptors in the brain reward circuits of human cocaine fatalities. J Neurosci, 1996:16:6100-6106. DOI: 10.1523/JNEUROSCI.16-19-06100.1996 (b) Segal DM, Moraes CT, Mash DC, Upregulation of D3 dopamine receptor mRNA in the nucleus accumbens of human cocaine fatalities. Brain Res Mol Brain Res 1997:45:335-339. DOI: 10.1016/s0169328x(97)00025-9 (c) Mash DC, Staley JK. D3 dopamine and kappa opioid receptor alterations in human brain of cocaine-overdose victims. Ann N Y Acad Sci, 1999:877:507-522. DOI: 10.1111/j.1749-6632.1999.tb09286.x

18. (a) Keck TM, John WS, Czoty PW, Nader MA, Newman AH. Identifying Medication Targets for Psychostimulant Addiction: Unraveling the Dopamine D3 Receptor Hypothesis. J Med Chem, 2015:58:5361-5380. DOI: 10.1021/jm501512b (b) Newman AH, Blaylock BL, Nader MA, Bergman J, Sibley DR, Skolnick P. Medication discovery 
for addiction: Translating the dopamine D3 receptor hypothesis. Biochem Pharmacol 2012:84:882-890. DOI:10.1016/j.bcp.2012.06.023 (c) Payer D, Balasubramaniam G. Boileau I, What is the role of the D3 receptor in addiction? A mini review of PET studies with [11C]-(+)-PHNO. Prog Neuropsychopharmacol Biol Psychiatry, 2014:52:4-8. DOI: 10.1016/j.pnpbp.2013.08.012 (d) Blaylock BL, Nader MA, Dopamine D3 Receptor Function and Cocaine Exposure, Neuropsychopharmacology, 2012:37:297-298. DOI: 10.1038/npp.2011.170

19. Millan MJ, Loiseau F, Dekeyne A, Gobert A. Flik G, Cremers, TI, Rivet JM, Sicard D, Billiras R, Brocco M. S33138 (N-[4-[2-[(3aS, 9bR)-8-cyano-1, 3a, 4, 9b-tetrahydro [1] benzopyrano [3, 4-c] pyrrol-2 (3H)-yl)-ethyl] phenyl-acetamide), a preferential dopamine D3 versus D2 receptor antagonist and potential antipsychotic agent: III. Actions in models of therapeutic activity and induction of side effects. J Pharmacol Exp Ther, 2008:324:3:1212-1226.

20. Chen PJ, Taylor M, Griffin SA, Amani A, Hayatshahi H, Korzekwa K, Ye M, Mach RH, Liu J, Luedtke RR, Gordon JC, Blass BE, Design, synthesis, and evaluation of N-(4-(4phenyl piperazin-1-yl)butyl)-4-(thiophen-3-yl)benzamides as selective dopamine D3 receptor ligands, Bioorg Med Chem Lett, 2019:29:18:2690-2694. DOI: 10.1016/j.bmcl.2019.07.020

21. Hayatshahi HS, Xu K, Griffin SA, Taylor M, Mach RH, Liu J, Luedtke RR. Analogues of Arylamide Phenylpiperazine Ligands To Investigate the Factors Influencing D3 Dopamine Receptor Bitropic Binding and Receptor Subtype Selectivity. ACS Chem Neurosci, 2018:9:2972-2983. DOI: 10.1021/acschemneuro.8b00142 